DAMTP-2013-50

\title{
The closed-string 3-loop amplitude and S-duality
}

\author{
Humberto Gomez ${ }^{\dagger}$ and Carlos R. Mafra ${ }^{\ddagger}$ \\ ${ }^{\dagger}$ Perimeter Institute for Theoretical Physics, Waterloo, Ontario N2L 2Y5, Canada \\ ${ }^{\dagger}$ Instituto de Física Teórica UNESP - Universidade Estadual Paulista \\ Caixa Postal 70532-2 01156-970 São Paulo, SP, Brazil \\ ${ }^{\ddagger} D A M T P$, University of Cambridge \\ Wilberforce Road, Cambridge, CB3 OWA, UK
}

\begin{abstract}
The low-energy limit of the four-point 3-loop amplitude (including its overall coefficient) is computed in both type IIA and IIB superstring theories using the pure spinor formalism. The result is shown to agree with the prediction of the coefficient for the type IIB $D^{6} R^{4}$ interaction made by Green and Vanhove based on S-duality considerations.
\end{abstract}

August 2013

${ }^{\dagger}$ email: humgomzu@ift.unesp.br

$\ddagger$ email: c.r.mafra@damtp.cam.ac.uk 


\section{Contents}

1 Introduction . . . . . . . . . . . . . . . . . . . . . . . . . . . . 2

2 Definitions and conventions . . . . . . . . . . . . . . . . . . . . . . . 3

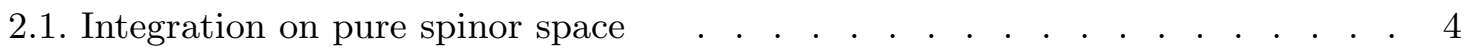

2.2. Four-point SYM amplitude and kinematics . . . . . . . . . . . . . . . 6

2.3. Riemann surfaces . . . . . . . . . . . . . . . . . . . . . . . . . . 7

2.4. The amplitude prescription . . . . . . . . . . . . . . . . . . . . . . 9

3 The closed-string 3-loop amplitude . . . . . . . . . . . . . . . . . . 10

3.1. $12 d_{\alpha}$ zero-modes from the $b$-ghosts . . . . . . . . . . . . . . . . . 10

3.2. $11 d_{\alpha}$ zero-modes from the $b$-ghosts . . . . . . . . . . . . . . . . . 12

3.3. The low-energy limit $D^{6} R^{4}$. . . . . . . . . . . . . . . . . . . . . 16

4 Perturbative calculations versus S-duality predictions _ . . . . . . 17

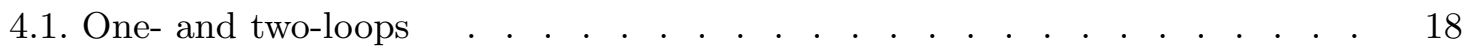

4.2. Three-loops . . . . . . . . . . . . . . . . . . . . . . . . . . . 19

A A general formula for integration of pure spinors . . . . . . . . . . 20

B Integration by parts . . . . . . . . . . . . . . . . . . . . . . . . . . 25 


\section{Introduction}

Up to this day superstring amplitudes in ten-dimensional Minkowski space have never been computed at genus higher than two. In this paper the low-energy limit of the genus three amplitude for four massless states in closed superstring theory is computed (including its overall coefficient) using the pure spinor formalism [1,2].

After the relatively straightforward pure spinor derivation of the two-loop amplitude ${ }^{1}$ in $[4,5]$, the natural question was how well the formalism would behave at higher genus. It is well-known by now that, in order to compute general amplitudes at genus higher than two the original BRST-invariant regulator of Berkovits [2] needs to be replaced by a more complicated scheme proposed by Berkovits and Nekrasov in [6]. Nevertheless, for four massless states at genus three one can still use the original regulator for the terms considered in this paper since they are F-terms and these were shown in [7] to be unaffected by the divergences which require the new regulator.

In addition to the regulator, there is one more point to consider though. As recently emphasized by Witten [8], to compute multiloop scattering amplitudes it is not sufficient to represent the external states by BRST-invariant vertex operators of definite conformal weight. The unintegrated vertex may have at most a simple pole singularity with the $b$ ghost while the integrated vertex must have no singularities at all. Unfortunately this is not the case for the massless pure spinor vertex operators of $[1,2]$ and one would probably need to use the vertices constructed in [9]. These vertices depend on the non-minimal variables and therefore require the concomitant use of the Berkovits-Nekrasov regulator.

Luckily, we will show that the low-energy limit (of order $D^{6} R^{4}$ ) of the genus-three amplitude is not affected by these considerations because only the zero modes of the $b$-ghost enter in the derivation. Any subtlety is deferred to terms of order $D^{8} R^{4}$ and higher.

With that in mind, one can proceed with the three-loop computation using the formalism as described in [2]. And ever since the normalizations for the pure spinor measures were determined in [10] and systematically used in [11], keeping track of the overall normalization does not pose additional difficulties. In doing so, the precise normalization of the amplitude at order $D^{6} R^{4}$ is shown to agree with the value predicted in 2005 by Green and Vanhove using S-duality arguments [12].

1 For the RNS derivation see the earlier works of D'Hoker and Phong, e.g. [3]. 


\section{Definitions and conventions}

The non-minimal pure spinor formalism action for the left-moving sector reads [2]

$$
S=\frac{1}{2 \pi \alpha^{\prime}} \int_{\Sigma_{g}} d^{2} z\left(\partial x^{m} \bar{\partial} x_{m}+\alpha^{\prime} p_{\alpha} \bar{\partial} \theta^{\alpha}-\alpha^{\prime} w_{\alpha} \bar{\partial} \lambda^{\alpha}-\alpha^{\prime} \bar{w}^{\alpha} \overline{\partial \lambda}_{\alpha}+\alpha^{\prime} s^{\alpha} \bar{\partial} r_{\alpha}\right),
$$

where $\lambda^{\alpha}$ and $\bar{\lambda}_{\beta}$ are bosonic pure spinors and $r_{\alpha}$ is a constrained fermionic variable,

$$
\left(\lambda \gamma^{m} \lambda\right)=0, \quad\left(\bar{\lambda} \gamma^{m} \bar{\lambda}\right)=0, \quad\left(\bar{\lambda} \gamma^{m} r\right)=0
$$

The fields in (2.1) have the following space-time dimensions [10]

$$
\left[\alpha^{\prime}\right]=2,\left[x^{m}\right]=1, \quad\left[\theta^{\alpha}, \lambda^{\alpha}, \bar{w}^{\alpha}, s^{\alpha}\right]=1 / 2, \quad\left[p_{\alpha}, w_{\alpha}, \bar{\lambda}_{\alpha}, r_{\alpha}\right]=-1 / 2 .
$$

The genus- $g$ OPEs for the matter variables following from (2.1) are [13]

$$
x^{m}(z, \bar{z}) x_{n}(w, \bar{w}) \sim \delta_{n}^{m} G(z, w), \quad p_{\alpha}(z) \theta^{\beta}(w) \sim \delta_{\alpha}^{\beta} \eta(z, w),
$$

where the Green's function $G(z, w)$ is written in terms of the prime form $E(z, w)$ and the global holomorphic 1-forms $w_{I}(z)$ as [14]

$$
G\left(z_{i}, z_{j}\right)=-\frac{\alpha^{\prime}}{2} \ln \left|E\left(z_{i}, z_{j}\right)\right|^{2}+\alpha^{\prime} \pi\left(\operatorname{Im} \int_{z_{i}}^{z_{j}} w_{I}\right)(\operatorname{Im} \Omega)_{I J}^{-1}\left(\operatorname{Im} \int_{z_{i}}^{z_{j}} w_{J}\right),
$$

and satisfies $\frac{2}{\alpha^{\prime}} \partial_{z_{i}} \bar{\partial}_{z_{j}} G\left(z_{i}, z_{j}\right)=2 \pi \delta^{(2)}\left(z_{i}-z_{j}\right)-\pi \Omega\left(z_{i}, z_{j}\right)$, where

$$
\Omega\left(z_{i}, z_{j}\right) \equiv \Omega_{i j} \equiv \sum_{I, J=1}^{3} w_{I}\left(z_{i}\right)(\operatorname{Im} \Omega)_{I J}^{-1} \bar{w}_{J}\left(\bar{z}_{j}\right)
$$

and $\Omega_{I J}$ is the period matrix which will be defined below. Furthermore,

$$
\eta\left(z_{i}, z_{j}\right)=\eta_{i j} \equiv-\frac{2}{\alpha^{\prime}} \frac{\partial}{\partial z_{i}} G\left(z_{i}, z_{j}\right) .
$$

The Green-Schwarz constraint $d_{\alpha}(z)$ and the supersymmetric momentum $\Pi^{m}(z)$ are

$$
d_{\alpha}=p_{\alpha}-\frac{1}{\alpha^{\prime}}\left(\gamma^{m} \theta\right)_{\alpha} \partial x_{m}-\frac{1}{4 \alpha^{\prime}}\left(\gamma^{m} \theta\right)_{\alpha}\left(\theta \gamma_{m} \partial \theta\right), \quad \Pi^{m}=\partial x^{m}+\frac{1}{2}\left(\theta \gamma^{m} \partial \theta\right)
$$

and satisfy the following OPEs [15]

$$
\begin{aligned}
d_{\alpha}(z) d_{\beta}(w) & \sim-\frac{2}{\alpha^{\prime}} \frac{\gamma_{\alpha \beta}^{m} \Pi_{m}}{z-w}, & d_{\alpha}(z) f(x(w), \theta(w)) & \sim \frac{D_{\alpha} f}{z-w}, \\
d_{\alpha}(z) \Pi^{m}(w) & \sim \frac{\gamma_{\alpha \beta}^{m} \partial \theta^{\beta}}{z-w}, & \Pi^{m}(z) f(x(w), \theta(w)) & \sim-\frac{\alpha^{\prime}}{2} \frac{k^{m} f}{z-w}
\end{aligned}
$$


where $D_{\alpha}=\frac{\partial}{\partial \theta^{\alpha}}+\frac{1}{2}\left(\gamma^{m} \theta\right)_{\alpha} k_{m}$ is the supersymmetric derivative and $f(x, \theta)$ represents a generic superfield. The $b$-ghost is given by [2] (see also $[16,17]$ )

$$
\begin{gathered}
b=s^{\alpha} \partial \bar{\lambda}_{\alpha}+\frac{1}{4(\lambda \bar{\lambda})}\left[2 \Pi^{m}\left(\bar{\lambda} \gamma_{m} d\right)-N_{m n}\left(\bar{\lambda} \gamma^{m n} \partial \theta\right)-J_{\lambda}(\bar{\lambda} \partial \theta)-\left(\bar{\lambda} \partial^{2} \theta\right)\right] \\
+\frac{\left(\bar{\lambda} \gamma^{m n p} r\right)}{192(\lambda \bar{\lambda})^{2}}\left[\frac{\alpha^{\prime}}{2}\left(d \gamma_{m n p} d\right)+24 N_{m n} \Pi_{p}\right]-\frac{\alpha^{\prime}}{2} \frac{\left(r \gamma_{m n p} r\right)}{16(\lambda \bar{\lambda})^{3}}\left[\left(\bar{\lambda} \gamma^{m} d\right) N^{n p}-\frac{\left(\bar{\lambda} \gamma^{p q r} r\right) N^{m n} N_{q r}}{8(\lambda \bar{\lambda})}\right],
\end{gathered}
$$

and satisfies $\{Q, b(z)\}=T(z)$ where the BRST charge $Q$ and the energy-momentum tensor $T(z)$ are

$$
Q=\oint\left(\lambda^{\alpha} d_{\alpha}+\bar{w}^{\alpha} r_{\alpha}\right), \quad T(z)=-\frac{1}{\alpha^{\prime}} \partial x^{m} \partial x_{m}-p_{\alpha} \partial \theta^{\alpha}+w_{\alpha} \partial \lambda^{\alpha}+\bar{w}^{\alpha} \partial \bar{\lambda}_{\alpha}-s^{\alpha} \partial r_{\alpha}
$$

From (2.3) it follows that $[Q]=[b]=[T]=0$.

The massless vertex operators are given by $V(z, \bar{z})=\kappa V(\theta) \otimes \tilde{V}(\bar{\theta}) e^{i k \cdot x}$ and $U(z, \bar{z})=$ $\kappa U(\theta) \otimes \tilde{U}(\bar{\theta}) e^{i k \cdot x}$, where

$$
V(z)=\lambda^{\alpha} A_{\alpha}, \quad U(z)=\partial \theta^{\alpha} A_{\alpha}+A_{m} \Pi^{m}+\frac{\alpha^{\prime}}{2} d_{\alpha} W^{\alpha}+\frac{\alpha^{\prime}}{4} N_{m n} \mathcal{F}^{m n}
$$

and $A_{\alpha}, A^{m}, W^{\alpha}, \mathcal{F}^{m n}$ are the $\mathcal{N}=1$ super-Yang-Mills superfields in ten dimensions satisfying [18]

$$
\begin{array}{ll}
D_{\alpha} A_{\beta}+D_{\beta} A_{\alpha}=\gamma_{\alpha \beta}^{m} A_{m}, & D_{\alpha} A_{m}=\left(\gamma_{m} W\right)_{\alpha}+k_{m} A_{\alpha} \\
D_{\alpha} \mathcal{F}_{m n}=2 k_{[m}\left(\gamma_{n]} W\right)_{\alpha}, & D_{\alpha} W^{\beta}=\frac{1}{4}\left(\gamma^{m n}\right)_{\alpha}{ }^{\beta} \mathcal{F}_{m n} .
\end{array}
$$

The space-time dimensions of the superfields and the vertex operators are

$$
\left[A_{\alpha}\right]=1 / 2, \quad\left[A_{m}\right]=0, \quad\left[W^{\alpha}\right]=-1 / 2, \quad\left[\mathcal{F}_{m n}\right]=-1, \quad[V(z)]=[U(z)]=1 .
$$

\subsection{Integration on pure spinor space}

The zero-mode measures for the non-minimal pure spinor variables in a genus- $g$ surface have space-time dimension zero and are given by $[2,10,11]$

$$
\begin{array}{ll}
{[d \lambda] T_{\alpha_{1} \ldots \alpha_{5}}=c_{\lambda} \epsilon_{\alpha_{1} \ldots \alpha_{16}} d \lambda^{\alpha_{6}} \ldots d \lambda^{\alpha_{16}}} & {[d w]=c_{w} T_{\alpha_{1} \ldots \alpha_{5}} \epsilon^{\alpha_{1} \ldots \alpha_{16}} d w_{\alpha_{6}} \ldots d w_{\alpha_{16}}} \\
{[d \bar{\lambda}] \bar{T}^{\alpha_{1} \ldots \alpha_{5}}=c_{\bar{\lambda}} \epsilon^{\alpha_{1} \ldots \alpha_{16}} d \bar{\lambda}_{\alpha_{6}} \ldots d \bar{\lambda}_{\alpha_{16}}} & {[d \bar{w}] T_{\alpha_{1} \ldots \alpha_{5}}=c_{\bar{w}} \epsilon_{\alpha_{1} \ldots \alpha_{16}} d \bar{w}^{\alpha_{6}} \ldots d \bar{w}^{\alpha_{16}}} \\
{[d r]=c_{r} \bar{T}^{\alpha_{1} \ldots \alpha_{5}} \epsilon_{\alpha_{1} \ldots \alpha_{16}} \partial_{r}^{\alpha_{6}} \ldots \partial_{r}^{\alpha_{16}}} & {\left[d s^{I}\right]=c_{s} T_{\alpha_{1} \ldots \alpha_{5}} \epsilon^{\alpha_{1} \ldots \alpha_{16}} \partial_{\alpha_{6}}^{s^{I}} \ldots \partial_{\alpha_{16}}^{s^{I}}} \\
{[d \theta]=c_{\theta} d^{16} \theta} & {\left[d d^{I}\right]=c_{d} d^{16} d^{I} .}
\end{array}
$$


The normalizations are

$$
\begin{aligned}
c_{\lambda} & =\left(\frac{\alpha^{\prime}}{2}\right)^{-2} \frac{1}{11 !}\left(\frac{A_{g}}{4 \pi^{2}}\right)^{11 / 2} & c_{w} & =\left(\frac{\alpha^{\prime}}{2}\right)^{2} \frac{(2 \pi)^{-11}}{11 ! 5 !} Z_{g}^{-11 / g} \\
c_{\bar{\lambda}} & =\left(\frac{\alpha^{\prime}}{2}\right)^{2} \frac{2^{6}}{11 !}\left(\frac{A_{g}}{4 \pi^{2}}\right)^{11 / 2} & c_{\bar{w}} & =\left(\frac{\alpha^{\prime}}{2}\right)^{-2} \frac{(\lambda \bar{\lambda})^{3}}{11 !(2 \pi)^{11}} Z_{g}^{-11 / g} \\
c_{r} & =\left(\frac{\alpha^{\prime}}{2}\right)^{-2} \frac{R}{11 ! 5 !}\left(\frac{2 \pi}{A_{g}}\right)^{11 / 2} & c_{s} & =\left(\frac{\alpha^{\prime}}{2}\right)^{2} \frac{(2 \pi)^{11 / 2} R^{-1}}{2^{6} 11 ! 5 !(\lambda \bar{\lambda})^{3}} Z_{g}^{11 / g} \\
c_{\theta} & =\left(\frac{\alpha^{\prime}}{2}\right)^{4}\left(\frac{2 \pi}{A_{g}}\right)^{16 / 2} & c_{d} & =\left(\frac{\alpha^{\prime}}{2}\right)^{-4}(2 \pi)^{16 / 2} Z_{g}^{16 / g} .
\end{aligned}
$$

where $A_{g}=\int d^{2} z \sqrt{g}$ is the area of the genus- $g$ Riemann surface and

$$
Z_{g}=\frac{1}{\sqrt{\operatorname{det}(2 \operatorname{Im} \Omega)}}, \quad g \geq 1
$$

The tensors $T_{\alpha_{1} \ldots \alpha_{5}}$ and $\bar{T}^{\alpha_{1} \ldots \alpha_{5}}$ in $(2.14)$,

$$
\begin{aligned}
T_{\alpha_{1} \alpha_{2} \alpha_{3} \alpha_{4} \alpha_{5}} & =\left(\lambda \gamma^{m}\right)_{\alpha_{1}}\left(\lambda \gamma^{n}\right)_{\alpha_{2}}\left(\lambda \gamma^{p}\right)_{\alpha_{3}}\left(\gamma_{m n p}\right)_{\alpha_{4} \alpha_{5}} \\
\bar{T}^{\alpha_{1} \alpha_{2} \alpha_{3} \alpha_{4} \alpha_{5}} & =\left(\bar{\lambda} \gamma^{m}\right)^{\alpha_{1}}\left(\bar{\lambda} \gamma^{n}\right)^{\alpha_{2}}\left(\bar{\lambda} \gamma^{p}\right)^{\alpha_{3}}\left(\gamma_{m n p}\right)^{\alpha_{4} \alpha_{5}}
\end{aligned}
$$

are totally antisymmetric due to the pure spinor constraint $(2.2)$ and satisfy $T \cdot \bar{T}=$ $5 ! 2^{6}(\lambda \bar{\lambda})^{3}$. As explained in [11], setting $R^{2}=\frac{\sqrt{2}}{2^{16} \pi}$ fixes the normalization of pure spinor tree-level amplitudes to be same as in the RNS computations of [19].

Using the above measures and the results of [10] one can show that the integration over an arbitrary number of pure spinors $\lambda^{\alpha}$ and $\bar{\lambda}_{\beta}$ is given by

$$
\int[d \lambda][d \bar{\lambda}] e^{-(\lambda \bar{\lambda})}(\lambda \bar{\lambda})^{m} \lambda^{\alpha_{1}} \cdots \lambda^{\alpha_{n}} \bar{\lambda}_{\beta_{1}} \cdots \bar{\lambda}_{\beta_{n}}=\left(\frac{A_{g}}{2 \pi}\right)^{11} \frac{12 \Gamma(8+m+n)}{\Gamma(11)} \mathcal{T}_{\beta_{1} \ldots \beta_{n}}^{\alpha_{1} \ldots \alpha_{n}}
$$

where $\mathcal{T}_{\beta_{1} \ldots \beta_{n}}^{\alpha_{1} \ldots \alpha_{n}}$ are the $\gamma$-matrix traceless tensors discussed in the Appendix $\mathrm{A}$ and $\Gamma(x)$ is the gamma function. Using $\mathcal{T}_{\alpha_{1} \ldots \alpha_{p}}^{\alpha_{1} \ldots \alpha_{p}}=1$ it follows that [11]

$$
\int[d \lambda][d \bar{\lambda}](\lambda \bar{\lambda})^{n} e^{-(\lambda \bar{\lambda})}=\left(\frac{A_{g}}{2 \pi}\right)^{11} \frac{\Gamma(8+n)}{7 ! 60} .
$$

For an arbitrary superfield $M(\lambda, \bar{\lambda}, \theta, r)$ we define

$$
\langle M(\lambda, \bar{\lambda}, \theta, r)\rangle_{(n, g)} \equiv \int[d \theta][d r][d \lambda][d \bar{\lambda}] \frac{\mathrm{e}^{-(\lambda \bar{\lambda})-(r \theta)}}{(\lambda \bar{\lambda})^{3-n}} M(\lambda, \bar{\lambda}, \theta, r)
$$


which implies in particular that

$$
\left\langle\left(\lambda^{3} \theta^{5}\right)\right\rangle_{(n, g)}=2^{7} R\left(\frac{2 \pi}{A_{g}}\right)^{5 / 2}\left(\frac{\alpha^{\prime}}{2}\right)^{2} \frac{\Gamma(8+n)}{7 !}\left\langle\left(\lambda^{3} \theta^{5}\right)\right\rangle,
$$

where $\left(\lambda^{3} \theta^{5}\right) \equiv\left(\lambda \gamma^{m} \theta\right)\left(\lambda \gamma^{n} \theta\right)\left(\lambda \gamma^{p} \theta\right)\left(\theta \gamma_{m n p} \theta\right)$ and the pure spinor bracket $\langle\ldots\rangle$ in the right-hand side is normalized as $\left\langle\left(\lambda^{3} \theta^{5}\right)\right\rangle=1[1]$. The subscript $g$ will be dropped whenever there is no chance for confusion.

From (2.9) and (2.8) follows that

$$
\Pi^{m}\left(z_{i}\right) \bar{\Pi}^{n}\left(\bar{z}_{j}\right) \sim \frac{\alpha^{\prime}}{2} \eta^{m n}\left(2 \pi \delta^{(2)}\left(z_{i}-z_{j}\right)-\pi \Omega\left(z_{i}, z_{j}\right)\right) .
$$

But using (2.22) directly leads to a mixing between left- and right-movers. Instead, one can keep the two sectors separate by expanding $\Pi^{m}(z)=\hat{\Pi}^{m}(z)+\sum_{I=1}^{g} \Pi_{I}^{m} w_{I}(z)$ and computing the holomorphic square with

$$
\Pi_{I}^{m} \bar{\Pi}_{J}^{n}=-\frac{\alpha^{\prime}}{2} \eta^{m n} \pi(\operatorname{Im} \Omega)_{I J}^{-1}
$$

Using this prescription, contributions containing a single $\Pi_{I}^{m}$ or $\bar{\Pi}_{I}^{m}$ vanish.

We use conventions where the (anti)symmetrization over $n$ indices includes a factor of $1 / n$ !, the generalized Kronecker delta is $\delta_{\beta_{1} \ldots \beta_{n}}^{\alpha_{1} \ldots \alpha_{n}} \equiv \delta_{\beta_{1}}^{\left[\alpha_{1}\right.} \cdots \delta_{\beta_{n}}^{\left.\alpha_{n}\right]}$ and satisfies $\delta_{\alpha_{1} \ldots \alpha_{n}}^{\alpha_{1} \ldots \alpha_{n}}=\left(\begin{array}{l}d \\ n\end{array}\right)$ where $d=10$ or $d=16$ for vector or spinor indices respectively. The integration over $\theta$ is given by $\int d^{16} \theta \theta^{\alpha_{1}} \ldots \theta^{\alpha_{16}}=\epsilon^{\alpha_{1} \ldots \alpha_{16}}$ and $\epsilon^{\alpha_{1} \ldots \alpha_{11} \sigma_{1} \ldots \sigma_{5}} \epsilon_{\alpha_{1} \ldots \alpha_{11} \beta_{1} \ldots \beta_{5}}=11 ! 5 ! \delta_{\beta_{1} \ldots \beta_{5}}^{\sigma_{1} \ldots \sigma_{5}}$.

The partition of 3-loop $d_{\alpha}$ zero-modes is denoted by $\left(N_{1}, N_{2}, N_{3}\right)_{d}$ and indicates that an expression contains $N_{I}$ factors of $d_{\alpha}^{I}$. Furthermore, we define

$$
\left(\epsilon \cdot T \cdot d^{I}\right) \equiv \epsilon^{\alpha_{1} \ldots \alpha_{16}} T_{\alpha_{1} \ldots \alpha_{5}} d_{\alpha_{6}}^{I} \cdots d_{\alpha_{16}}^{I}, \quad\left(\bar{\lambda} r d^{I} d^{J}\right) \equiv\left(\bar{\lambda} \gamma^{m n p} r\right)\left(d^{I} \gamma_{m n p} d^{J}\right) .
$$

Two integrals frequently used in the next sections are summarized here,

$$
\begin{gathered}
\int\left[d d^{I}\right]\left(\epsilon \cdot T \cdot d^{I}\right) d_{\alpha_{1}}^{I} d_{\alpha_{2}}^{I} d_{\alpha_{3}}^{I} d_{\alpha_{4}}^{I} d_{\alpha_{5}}^{I}=11 ! 5 ! c_{d} T_{\alpha_{1} \alpha_{2} \alpha_{3} \alpha_{4} \alpha_{5}} \\
\int\left[d d^{I}\right]\left(\epsilon \cdot T \cdot d^{I}\right) d_{\alpha_{1}}^{I} d_{\alpha_{2}}^{I} d_{\alpha_{3}}^{I}\left(d^{I} \gamma^{m n p} d^{I}\right)=11 ! 5 ! 96 c_{d}\left(\lambda \gamma^{[m}\right)_{\alpha_{1}}\left(\lambda \gamma^{n}\right)_{\alpha_{2}}\left(\lambda \gamma^{p]}\right)_{\alpha_{3}} .
\end{gathered}
$$

\subsection{Four-point SYM amplitude and kinematics}

In $[19,11]$ the amplitudes in the Neveu-Schwarz (NS) sector were written using the kinematic factor $K$ defined as

$$
\begin{aligned}
K & =F_{1}^{m n} F_{2}^{n m} F_{3}^{p q} F_{4}^{q p}+F_{1}^{m n} F_{3}^{n m} F_{2}^{p q} F_{4}^{q p}+F_{1}^{m n} F_{4}^{n m} F_{2}^{p q} F_{3}^{q p} \\
& -4\left(F_{1}^{m n} F_{2}^{n p} F_{3}^{p q} F_{4}^{q m}+F_{1}^{m n} F_{3}^{n p} F_{2}^{p q} F_{4}^{q m}+F_{1}^{m n} F_{2}^{n p} F_{4}^{p q} F_{3}^{q m}\right)
\end{aligned}
$$


where $F_{m n}=k_{m} e_{n}-k_{n} e_{m}$ is the field-strength. Since the amplitudes in the pure spinor formalism are manifestly supersymmetric one can rewrite $K$ as follows

$$
K=-2^{3} 2880 A_{1234}^{\mathrm{YM}} s_{12} s_{23}
$$

where $A_{1234}^{\mathrm{YM}}$ is the ten-dimensional SYM amplitude normalized as $A_{1234}^{\mathrm{YM}}=\left\langle V_{1} E_{234}\right\rangle[20]$ and $s_{i j}=k^{i} \cdot k^{j}$ are the Mandelstam invariants. Furthermore $k^{i} \cdot k^{i}=0$ is the massless condition and $k_{m}^{1}+\cdots+k_{m}^{4}=0$ is the momentum conservation relation. In order to keep the momentum expansion formulæ of section 4 legible, we use the following definitions [21],

$$
\sigma_{2}=\left(\frac{\alpha^{\prime}}{2}\right)^{2}\left(s_{12}^{2}+s_{13}^{2}+s_{14}^{2}\right), \quad \sigma_{3}=\left(\frac{\alpha^{\prime}}{2}\right)^{3}\left(s_{12}^{3}+s_{13}^{3}+s_{14}^{3}\right)
$$

and note that $\sigma_{3}=3\left(\alpha^{\prime} / 2\right)^{3} s_{12} s_{13} s_{14}$.

\subsection{Riemann surfaces}

A holomorphic field with conformal weight one in a genus- $g$ Riemann surface $\Sigma_{g}$ can be expanded in a basis of holomorphic one-forms as $\phi(z)=\hat{\phi}(z)+\sum_{I=1}^{g} w_{I}(z) \phi^{I}$ and $\phi^{I}$ are the zero modes of $\phi(z)$. If $\left\{a_{I}, b_{J}\right\}$ are the generators of the $H_{1}\left(\Sigma_{g}, \mathbb{Z}\right)=\mathbb{Z}^{2 g}$ homology group, the holomorphic one-forms can be chosen such that for $I, J=1,2, \ldots, g$

$$
\int_{a_{I}} w_{J}(z) d z=\delta_{I J}, \quad \int_{b_{I}} w_{J}(z) d z=\Omega_{I J}, \quad \int_{\Sigma_{g}} w_{I} \bar{w}_{J} d^{2} z=2 \operatorname{Im} \Omega_{I J}
$$

where $\Omega_{I J}$ is the symmetric period matrix with $g(g+1) / 2$ complex degrees of freedom [22] and $d^{2} z=i d z \wedge d \bar{z}=2 d \operatorname{Re}(z) d \operatorname{Im}(z)$. For the three-loop amplitude we define

$$
\begin{aligned}
\Delta\left(z_{i} ; z_{j} ; z_{k}\right) & \equiv \epsilon^{I J K} w_{I}\left(z_{i}\right) w_{J}\left(z_{j}\right) w_{K}\left(z_{k}\right), \\
\Delta^{m}\left(z_{i}, z_{j} ; z_{k} ; z_{l}\right) & \equiv \epsilon^{I J K}(\Pi w)_{I}^{m}\left(z_{i}, z_{j}\right) w_{J}\left(z_{k}\right) w_{K}\left(z_{l}\right),
\end{aligned}
$$

where $(\Pi w)_{I}^{m}\left(z_{i}, z_{j}\right) \equiv \Pi_{I}^{m} w_{I}\left(z_{i}\right) w_{I}\left(z_{j}\right)$, (no sum in I). It follows that $\Delta^{m}\left(z_{i}, z_{j} ; z_{k} ; z_{l}\right)$ is symmetric in $(i j)$ and antisymmetric in $[k l]$ and satisfies

$$
\Pi_{I}^{m} w_{I}\left(z_{i}\right) \Delta\left(z_{j} ; z_{k} ; z_{l}\right)=\Delta^{m}\left(z_{i}, z_{j} ; z_{k} ; z_{l}\right)+\Delta^{m}\left(z_{i}, z_{k} ; z_{l} ; z_{j}\right)+\Delta^{m}\left(z_{i}, z_{l} ; z_{j} ; z_{k}\right)
$$

Furthermore, the period matrix extends a lattice called the Jacobian variety $[14,22], J=$ $\mathbb{C}^{g} /\left(\mathbb{Z}^{g}+\Omega \mathbb{Z}^{g}\right)$, which is invariant under the modular group $S p(2 g, \mathbb{Z})$. And finally, we define

$$
\int_{\Sigma_{4}} \equiv \int \prod_{i=1}^{4} d^{2} z_{i}
$$




\subsubsection{Moduli space}

The moduli space $\mathcal{M}_{g}$ is defined as the space of inequivalent complex structures on the Riemann surface $\Sigma_{g}$. It is well known that its complex $\operatorname{dimension}$ is $\operatorname{dim}_{\mathbb{C}}\left(\mathcal{M}_{g}\right)=3 g-3$, for $g>1$. We denote the complex coordinates on this space by $\tau_{i}$ for $i=1, \ldots, 3 g-3$.

For genus two and three the dimension of the moduli space is the same as the dimension of the period matrices, i.e., $3 g-3=g(g+1) / 2$ for $g=2$, 3. So there is a one-to-one map between inequivalent complex structures and inequivalent period matrices. This means that for genus $g=2,3$ the scattering amplitude can be written in terms of the period matrix instead of the moduli coordinates and Beltrami differentials. This rewriting can be achieved using the identities [14]

$$
\int d^{2} z w_{I}(z) w_{J}(z) \mu_{i}(z)=\frac{\delta \Omega_{I J}}{\delta \tau_{i}}, \quad \int \prod_{j=1}^{6} d^{2} \tau_{j}\left|\epsilon_{i_{1} \ldots i_{6}} \frac{\delta \Omega_{11}}{\delta \tau_{i_{1}}} \ldots \frac{\delta \Omega_{33}}{\delta \tau_{i_{6}}}\right|^{2}=\int \prod_{I \leq J}^{3} d^{2} \Omega_{I J}
$$

where the Beltrami differential is given by $\mu_{i z}^{\bar{z}}=\partial_{z} v_{i}^{\bar{z}}$ and $v_{i}^{\bar{z}}(z, \bar{z})$ is a small complex structure deformation.

However the factor $\prod_{I \leq J}^{3} d^{2} \Omega_{I J}$ is not invariant under the modular transformation $S p(6, \mathbb{Z})$. In general, the $S p(2 g, \mathbb{Z})$-invariant measure for the genus- $g$ moduli space is

$$
d \mu_{g} \equiv \frac{d^{2} \Omega_{I J}}{\left(\operatorname{det} \operatorname{Im} \Omega_{I J}\right)^{g+1}}
$$

and this is precisely the measure that will be obtained from first principles in the next section for genus $g=3$.

The corresponding volume of the inequivalent period matrix space is given in [23]

$$
\mathrm{Vol}_{g} \equiv \int_{\mathcal{M}_{g}} d \mu_{g}=2^{g^{2}+1}(2 \pi)^{g(g+1) / 2} \prod_{k=1}^{g} \frac{(k-1) !}{(2 k) !}\left|B_{2 k}\right|
$$

where $B_{2 k}$ are the Bernoulli numbers and the extra $2^{g(g+1) / 2}$ factor in (2.35) compared to the original formula in [23] is due to a different convention for $d^{2} \Omega_{I J}$ (see e.g. [19]). In particular,

$$
\mathrm{Vol}_{1}=\frac{2 \pi}{3}, \quad \mathrm{Vol}_{2}=\frac{4 \pi^{3}}{3^{3} 5}, \quad \mathrm{Vol}_{3}=\frac{2^{6} \pi^{6}}{3^{6} 5^{2} 7}
$$




\subsection{The amplitude prescription}

The prescription to compute the multiloop $n$-point closed-string amplitude was given in $[2]$ and it becomes ${ }^{2}$.

$$
\mathcal{A}_{3}=\kappa^{4} e^{4 \lambda} \int_{\mathcal{M}_{3}} \prod_{j=1}^{6} d^{2} \tau_{j} \int_{\Sigma_{4}}\left|\left\langle\mathcal{N}\left(b, \mu_{j}\right) U^{1}\left(z_{1}\right) \ldots U^{4}\left(z_{4}\right)\right\rangle\right|^{2}
$$

for three loops and four points. $\mathcal{M}_{3}$ is the fundamental domain of the genus-three Riemann surface. The $b$-ghost insertion is

$$
\left(b, \mu_{j}\right)=\frac{1}{2 \pi} \int d^{2} y_{j} b_{z z} \mu_{j \bar{z}}^{z}, \quad j=1, \ldots, 6 .
$$

After the non-zero modes are integrated out using their OPEs, the pure spinor bracket $\langle\ldots\rangle$ denotes the integration over the zero-modes ${ }^{3}$

$$
\langle\ldots\rangle=\int[d \theta][d r][d \lambda][d \bar{\lambda}] \prod_{I=1}^{3}\left[d d^{I}\right]\left[d s^{I}\right]\left[d \bar{w}^{I}\right]\left[d w^{I}\right]
$$

and $\mathcal{N}$ is the BRST regulator discussed in [2] which can be written as

$$
\mathcal{N}=\sum_{I=1}^{3} e^{-(\lambda \bar{\lambda})-\left(w^{I} \bar{w}^{I}\right)-(r \theta)+\left(s^{I} d^{I}\right)}
$$

After the integration over $\left[d d^{I}\right]\left[d s^{I}\right]\left[d w^{I}\right]\left[d \bar{w}^{I}\right]$ is performed, the remaining variables $\lambda^{\alpha}, \bar{\lambda}_{\beta}, \theta^{\delta}$ and $r_{\alpha}$ have conformal weight zero and therefore are the same ones which need to be integrated in the prescription of the tree-level amplitudes. Using the Theorem 1 from Appendix A all correlators at this stage of the computation reduce to pure spinor superspace expressions [24] whose component expansions can be straightforwardly computed $[25,26]$. In particular, the last correlator to evaluate is a combination of the zero mode integration of tree-level pure spinor variables $(2.21)$ and $x^{m}$,

$$
\left|\left\langle\left(\lambda^{3} \theta^{5}\right)\right\rangle_{(n, g)}\right|^{2}\left\langle\prod_{j=1}^{4} e^{i k^{j} \cdot x^{j}}\right\rangle=(2 \pi)^{10} \delta^{(10)}(k) \frac{\sqrt{2}}{2^{7} \pi^{6}}\left(\frac{\alpha^{\prime}}{2}\right)^{-1}\left(\frac{\Gamma(8+n)}{7 !}\right)^{2}\left|\left\langle\left(\lambda^{3} \theta^{5}\right)\right\rangle\right|^{2} \mathcal{I}\left(s_{i j}\right),
$$

2 In the first version of this paper, we argued for an overall symmetry factor $1 / 3$ for the threeloop amplitude. We thank Edward Witten for explaining to us that such factor was incorrect.

3 The definition of the pure spinor bracket here should not be confused with the standard zero mode integration $\left\langle\left(\lambda^{3} \theta^{5}\right)\right\rangle=1$ of [1]. Since the context makes the distinction clear, we chose not to distinguish the notation. 
where $\delta^{(10)}(k) \equiv \delta^{(10)}\left(\sum_{i} k_{i}^{m}\right)$ and $\mathcal{I}\left(s_{i j}\right)$ is the Koba-Nielsen factor

$$
\mathcal{I}\left(s_{i j}\right)=\exp \left(-\sum_{i<j} s_{i j} G\left(z_{i}, z_{j}\right)\right)
$$

Given the above conventions, the space-time dimension of the closed-string $n$-point amplitude is independent of the genus; $\left[\mathcal{A}_{g}\right]=n(2+[\kappa])$. One can show that unitarity [27] requires $\kappa^{2} e^{-2 \lambda}=\left(\alpha^{\prime} / 2\right)^{-2} \sqrt{2} 2^{8} \pi^{7}$, so $[\kappa]=-2$ and the amplitudes are dimensionless.

\section{The closed-string 3-loop amplitude}

At genus three there are $(16,16,16)_{d}$ zero-modes of $d_{\alpha}$ and $(11,11,11)_{s}$ zero-modes of $s^{\alpha}$. The factor $e^{\left(d^{I} s^{I}\right)}$ in the regulator $(2.40)$ is the only source of $s^{\alpha}$ zero-modes so the integration over $\left[d s^{I}\right]$ brings down $(11,11,11)_{d}$ zero-modes,

$$
\int \prod_{I=1}^{3}\left[d s^{I}\right] e^{-\left(d^{I} s^{I}\right)}=\left(\frac{\alpha^{\prime}}{2}\right)^{6} \frac{(2 \pi)^{33 / 2} Z_{3}^{11}}{R^{3} 2^{18}(11 ! 5 !)^{3}(\lambda \bar{\lambda})^{9}} \prod_{I=1}^{3}\left(\epsilon \cdot T \cdot d^{I}\right) .
$$

The remaining $(5,5,5)_{d}$ must come from the $b$-ghosts and the external vertices. Since the number of $d_{\alpha}$ zero-modes from the external vertices and from each $b$-ghost can be at most four and two respectively, there are only two possibilities for the $b$-ghosts: they provide 11 or $12 d_{\alpha}$ zero modes. Note that these possibilities lead to integrations over pure spinor variables which can be regularized using the original procedure of Berkovits [2].

In the following we decompose the amplitude (2.37) according to the two different b-ghost sectors as $\mathcal{A}_{3}=A_{11}+A_{12}$ and evaluate each sector in turn.

\section{1. $12 d_{\alpha}$ zero-modes from the b-ghosts}

In this sector there is no chance for OPE singularities between the $b$-ghosts and the external vertices and therefore $(b, \mu)$ is still a well-defined measure [8]. To see this note that if six $b$-ghosts provide twelve $d_{\alpha}$ zero modes, each one of them must pick the term $\left(\bar{\lambda} \gamma^{m n p} r\right)\left(d \gamma_{m n p} d\right) /\left(192(\lambda \bar{\lambda})^{2}\right)$ in $(2.10)$. The zero-mode part of each $\left(d \gamma_{m n p} d\right)(y)$ factor is

$$
\begin{array}{r}
\left(d^{1} \gamma_{m n p} d^{1}\right) w_{1}(y) w_{1}(y)+2\left(d^{1} \gamma_{m n p} d^{2}\right) w_{1}(y) w_{2}(y)+2\left(d^{1} \gamma_{m n p} d^{3}\right) w_{1}(y) w_{3}(y) \\
+\left(d^{2} \gamma_{m n p} d^{2}\right) w_{2}(y) w_{2}(y)+2\left(d^{2} \gamma_{m n p} d^{3}\right) w_{2}(y) w_{3}(y)+\left(d^{3} \gamma_{m n p} d^{3}\right) w_{3}(y) w_{3}(y)
\end{array}
$$


and a short computation using (2.33) gives,

$$
\int \prod_{j=1}^{6} d^{2} \tau_{j}\left|\left(b, \mu_{j}\right)\right|^{2}=c_{b_{1}}^{2} \int d^{2} \Omega_{I J}\left|\frac{B_{(4,4,4)}}{(\lambda \bar{\lambda})^{12}}\right|^{2}
$$

where $c_{b_{1}}=\left(\frac{\alpha^{\prime}}{2}\right)^{6} \frac{2^{3}}{\left(2^{7} 3 \pi\right)^{6}}$ and

$$
B_{(4,4,4)} \equiv\left(\bar{\lambda} r d^{1} d^{1}\right)\left(\bar{\lambda} r d^{1} d^{2}\right)\left(\bar{\lambda} r d^{1} d^{3}\right)\left(\bar{\lambda} r d^{2} d^{2}\right)\left(\bar{\lambda} r d^{2} d^{3}\right)\left(\bar{\lambda} r d^{3} d^{3}\right)
$$

Note that $B_{(4,4,4)}$ is totally symmetric in the zero-mode labels (123). Since $w^{I}$ and $\bar{w}^{I}$ appear only in the regulator $\mathcal{N}$ their integration is straightforward

$$
\int \prod_{I=1}^{3}\left[d w^{I}\right]\left[d \bar{w}^{I}\right] \mathrm{e}^{-\left(w^{I} \bar{w}^{I}\right)}=\frac{(\lambda \bar{\lambda})^{9}}{(2 \pi)^{33}} Z_{3}^{-22}
$$

Defining (if $B_{(p, q, r)}$ does not contain an index $m$ one omits it altogether on both sides)

$$
D_{(p+11, q+11, r+11)}^{m} \equiv \int \prod_{I=1}^{3}\left[d d^{I}\right]\left(\epsilon \cdot T \cdot d^{I}\right) B_{(p, q, r)}^{m}
$$

and gathering the above results,

$$
A_{12}=\frac{\sqrt{2} 2^{-101} \kappa^{4} e^{4 \lambda}}{\pi^{42} 3^{12}(11 ! 5 !)^{6}}\left(\frac{\alpha^{\prime}}{2}\right)^{24} \int \frac{d^{2} \Omega_{I J}}{Z_{3}^{22}} \int_{\Sigma_{4}}\left|\left\langle D_{(15,15,15)} U^{1} U^{2} U^{3} U^{4}\right\rangle_{(-9)}\right|\left\langle\prod_{j=1}^{2} \mathrm{e}^{i k^{j} \cdot x^{j}}\right\rangle .
$$

The only non-vanishing contribution to the integral in (3.6) contains three $d_{\alpha}$ from the external vertices,

$$
\begin{aligned}
U_{1} U_{2} U_{3} U_{4}=\left(\frac{\alpha^{\prime}}{2}\right)^{4}\left[\left(d W_{12}\right)\left(d W_{3}\right)\left(d W_{4}\right) \eta_{12}+\left(d W_{13}\right)\left(d W_{2}\right)\left(d W_{4}\right) \eta_{13}\right. \\
+\left(d W_{14}\right)\left(d W_{2}\right)\left(d W_{3}\right) \eta_{14}+\left(d W_{23}\right)\left(d W_{1}\right)\left(d W_{4}\right) \eta_{23} \\
\left.+\left(d W_{24}\right)\left(d W_{1}\right)\left(d W_{3}\right) \eta_{24}+\left(d W_{34}\right)\left(d W_{1}\right)\left(d W_{2}\right) \eta_{34}\right] \\
+\left(\frac{\alpha^{\prime}}{2}\right)^{3} \sum_{I=1}^{3} \Pi_{I}^{m} w_{I}\left(z_{1}\right) A_{m}^{1}\left(d W^{2}\right)\left(d W^{3}\right)\left(d W^{4}\right)+(1 \leftrightarrow 2,3,4)
\end{aligned}
$$

where $W_{i j}$ denotes the BRST block [28],

$$
W_{i j}^{\alpha}=\frac{1}{4}\left(\gamma_{p q} W_{j}\right)^{\alpha} \mathcal{F}_{i}^{p q}+\left(k_{j} \cdot A_{i}\right) W_{j}^{\alpha}-(i \leftrightarrow j)
$$


Since now only the zero modes contribute, each $d_{\alpha}\left(z_{i}\right)$ becomes $d_{\alpha}^{I} w_{I}\left(z_{i}\right)$. Note that $D_{(15,15,15)} d_{\alpha}^{I} d_{\beta}^{J} d_{\gamma}^{K} w_{I}\left(z_{i}\right) w_{J}\left(z_{j}\right) w_{K}\left(z_{k}\right)=D_{(15,15,15)} d_{\alpha}^{1} d_{\beta}^{2} d_{\gamma}^{3} \Delta\left(z_{i} ; z_{j} ; z_{k}\right)$ because the only non-vanishing contribution has $(1,1,1)_{d}$ zero-modes and $\prod_{I=1}^{3}\left[d d^{I}\right]\left(\epsilon \cdot T \cdot d^{I}\right)$ is totally antisymmetric in the zero-mode labels [123]. Thus,

$$
\begin{aligned}
D_{(15,15,15)}\left(d W_{i j}\right)\left(d W_{k}\right)\left(d W_{l}\right) & =(11 ! 5 !)^{3} 96^{3} c_{d}^{3} T_{i j, k, l}(\lambda, \bar{\lambda}, r) \Delta\left(z_{j} ; z_{k} ; z_{l}\right) \\
D_{(15,15,15)}\left(d W_{i}\right)\left(d W_{j}\right)\left(d W_{k}\right) A_{l}^{m} & =(11 ! 5 !)^{3} 96^{3} c_{d}^{3} L_{i j k l}^{m}(\lambda, \bar{\lambda}, r) \Delta\left(z_{j} ; z_{k} ; z_{l}\right)
\end{aligned}
$$

where

$$
\begin{aligned}
T_{i j, k, l}(\lambda, \bar{\lambda}, r)= & \left(\bar{\lambda} \gamma^{a b c} r\right)\left(\bar{\lambda} \gamma^{\text {def }} r\right)\left(\bar{\lambda} \gamma^{g h i} r\right)\left(\bar{\lambda} \gamma^{m n p} r\right)\left(\bar{\lambda} \gamma^{q r s} r\right)\left(\bar{\lambda} \gamma^{t u v} r\right) \\
& \times\left(\lambda \gamma^{a d e f m} \lambda\right)\left(\lambda \gamma^{b g h i t} \lambda\right)\left(\lambda \gamma^{u q r s n} \lambda\right)\left(\lambda \gamma^{c} W_{i j}\right)\left(\lambda \gamma^{p} W_{k}\right)\left(\lambda \gamma^{v} W_{l}\right) \\
L_{i j k l}^{m}(\lambda, \bar{\lambda}, r)= & \left(\bar{\lambda} \gamma^{a b c} r\right)\left(\bar{\lambda} \gamma^{\text {def }} r\right)\left(\bar{\lambda} \gamma^{g h i} r\right)\left(\bar{\lambda} \gamma^{m n p} r\right)\left(\bar{\lambda} \gamma^{q r s} r\right)\left(\bar{\lambda} \gamma^{t u v} r\right) \\
& \times\left(\lambda \gamma^{a d e f m} \lambda\right)\left(\lambda \gamma^{b g h i t} \lambda\right)\left(\lambda \gamma^{u q r s n} \lambda\right)\left(\lambda \gamma^{c} W_{i}\right)\left(\lambda \gamma^{p} W_{j}\right)\left(\lambda \gamma^{v} W_{k}\right) A_{l}^{m} .
\end{aligned}
$$

As shown in the Appendix A, it is always possible to rewrite the $\bar{\lambda}^{n} \lambda^{n+3}$ dependence in (3.10) as $(\lambda \bar{\lambda})^{n} \lambda^{3}$ when performing the zero mode integrals and therefore we write $T_{i j, k, l}(\lambda, \bar{\lambda}, r)=(\lambda \bar{\lambda})^{6} T_{i j, k, l}(\lambda, r)$ and drop the $(\lambda, r)$ arguments from now on. Note that $T_{i j, k, l}$ is antisymmetric in $[i j]$ and $[k l]$ and $L_{i j k l}^{m}$ is antisymmetric in $[i j k]$.

Plugging the above results in (3.6) and using the identity (2.31) together with the definitions $M_{i j, k, l}=s_{i j}^{-1} T_{i j, k, l}, X_{i j}=\left(\alpha^{\prime} / 2\right) s_{i j} \eta_{i j}$ yields

$$
A_{12}=\frac{\sqrt{2} \pi^{6}}{2^{23} 3^{6}} \kappa^{4} e^{4 \lambda}\left(\frac{\alpha^{\prime}}{2}\right)^{6} \int \frac{d^{2} \Omega_{I J}}{Z_{3}^{-10}} \int_{\Sigma_{4}}\left\langle\left|\mathcal{K}_{12}\right|^{2}\right\rangle_{(-3)}\left\langle\prod_{j=1}^{4} \mathrm{e}^{i k^{j} \cdot x^{j}}\right\rangle
$$

where

$$
\begin{aligned}
\mathcal{K}_{12} & =M_{12,3,4} \Delta\left(z_{2} ; z_{3} ; z_{4}\right) X_{12}+M_{13,2,4} \Delta\left(z_{3} ; z_{2} ; z_{4}\right) X_{13}+M_{14,2,3} \Delta\left(z_{4} ; z_{2} ; z_{3}\right) X_{14} \\
& +M_{23,1,4} \Delta\left(z_{3} ; z_{1} ; z_{4}\right) X_{23}+M_{24,1,3} \Delta\left(z_{4} ; z_{1} ; z_{3}\right) X_{24}+M_{34,1,2} \Delta\left(z_{4} ; z_{1} ; z_{2}\right) X_{34} \\
& +\Delta^{m}\left(z_{1}, z_{2} ; z_{3} ; z_{4}\right)\left[L_{1342}^{m}+L_{2341}^{m}\right]+\Delta^{m}\left(z_{1}, z_{3} ; z_{2} ; z_{4}\right)\left[L_{1243}^{m}+L_{3241}^{m}\right] \\
& +\Delta^{m}\left(z_{1}, z_{4} ; z_{2} ; z_{3}\right)\left[L_{1234}^{m}+L_{4231}^{m}\right]+\Delta^{m}\left(z_{2}, z_{3} ; z_{1} ; z_{4}\right)\left[L_{2143}^{m}+L_{3142}^{m}\right] \\
& +\Delta^{m}\left(z_{2}, z_{4} ; z_{1} ; z_{3}\right)\left[L_{2134}^{m}+L_{4132}^{m}\right]+\Delta^{m}\left(z_{3}, z_{4} ; z_{1} ; z_{2}\right)\left[L_{3124}^{m}+L_{4123}^{m}\right]
\end{aligned}
$$

\section{2. $11 d_{\alpha}$ zero-modes from the b-ghosts}

By not using the Siegel-gauge vertex operators of [9] one could in principle face problems with the consistency condition for $(b, \mu)$ discussed in [8]. However, in the low-energy limit 
discussed here, this potential complication can be ignored since the only contribution comes from terms in which the b ghost does not have singular $\mathrm{OPEs}^{4}$. To see this note that one possibility to obtain $11 d_{\alpha}$ zero modes out of six $b$-ghosts is given by

$$
b^{6} \longrightarrow \frac{(\lambda \bar{\lambda})^{-12}}{(192)^{6}}\left(\frac{\alpha^{\prime}}{2}\right)^{6}\left[\left(\bar{\lambda} \gamma^{m n p} r\right)\left(d \gamma_{m n p} d\right)\right]^{6}
$$

where one of the $d_{\alpha}\left(y_{i}\right)$ is the non-zero-mode part $\hat{d}_{\alpha}\left(y_{i}\right)$ and contracts through the OPE (2.9) with an external vertex ( $y_{i}$ denotes the position of the corresponding $b$-ghost). However this term is of order $D^{8} R^{4}$ and there are no inverse powers of Mandelstam invariants coming from the integration over the vertex positions since there are no simple pole singularities among them [29], as they must contribute the four remaining $d_{\alpha}$ zero modes. The claim that (3.13) leads to terms of order $D^{8} R^{4}$ is easy to verify. The external vertices contribute $W^{4}$ superfields, the OPE between $\hat{d}_{\alpha}(y)$ and one superfield $W^{\beta}$ gives $D_{\alpha} W^{\beta}$ and each $r_{\alpha}$ from (3.13) counts as a covariant derivative $D_{\alpha}$ because of the factor $e^{-(r \theta)}$ in the regulator $\mathcal{N}$. This gives kinematic terms proportional to $\left\langle D_{\alpha}^{7} W^{4}\right\rangle=\left\langle k^{3} W^{3} \mathcal{F}\right\rangle=k^{4} F_{m n}^{4}$ whose holomorphic square is $D^{8} R^{4}$. The other possibilities of $b$-ghost singularities are similarly analyzed. Therefore the terms which might be affected by the issues pointed out in [8] do not affect the leading order terms $D^{6} R^{4}$ and will not be considered in the following.

When the $b$-ghosts have no singularities with the vertices and contribute 11 zero-modes of $d_{\alpha}$, one possibility is

$$
b^{6} \longrightarrow \frac{(\lambda \bar{\lambda})^{-13}}{16(192)^{5}}\left(\frac{\alpha^{\prime}}{2}\right)^{6}\left(r \gamma_{q r s} r\right)\left(\bar{\lambda} \gamma^{q} d\right) N^{r s}\left[\left(\bar{\lambda} \gamma^{m n p} r\right)\left(d \gamma_{m n p} d\right)\right]^{5}
$$

but it vanishes upon integration over $[d w]$ because $\int[d w][d \bar{w}] w_{\alpha} e^{-\left(w^{I} \bar{w}_{I}\right)}=0$. The other possibility is

$$
b^{6} \longrightarrow \frac{(\lambda \bar{\lambda})^{-11}}{2(192)^{5}}\left(\frac{\alpha^{\prime}}{2}\right)^{5}\left(\bar{\lambda} \gamma_{q} d\right) \Pi^{q}\left[\left(\bar{\lambda} \gamma^{m n p} r\right)\left(d \gamma_{m n p} d\right)\right]^{5}
$$

where the $\Pi^{m}(y)$ field is proportional to its zero modes $\Pi_{m}^{I} w_{I}(y)$. In this case the integration over the positions of the $b$-ghosts can be carried out,

$$
\int \prod_{j=1}^{6} d^{2} \tau_{j}\left|\left(b, \mu_{j}\right)\right|^{2}=c_{b_{2}}^{2} \int d^{2} \Omega_{I J}\left|\frac{1}{(\lambda \bar{\lambda})^{11}}\left(\Pi_{m}^{1} B_{(3,4,4)}^{m}+\Pi_{m}^{2} B_{(4,3,4)}^{m}+\Pi_{m}^{3} B_{(4,4,3)}^{m}\right)\right|^{2}
$$

4 We are grateful to Nathan Berkovits for discussions and for his comments on the draft at this point. 
where $c_{b_{2}}=\frac{2^{2}\left(\alpha^{\prime} / 2\right)^{5}}{4 \pi\left(2^{7} 3 \pi\right)^{5}}=48\left(\alpha^{\prime} / 2\right)^{-1} c_{b_{1}}$ and

$$
\begin{aligned}
B_{(3,4,4)}^{m}= & +2\left(\bar{\lambda} \gamma^{m} d^{1}\right)\left(\bar{\lambda} r d^{1} d^{2}\right)\left(\bar{\lambda} r d^{1} d^{3}\right)\left(\bar{\lambda} r d^{2} d^{2}\right)\left(\bar{\lambda} r d^{2} d^{3}\right)\left(\bar{\lambda} r d^{3} d^{3}\right) \\
& -\left(\bar{\lambda} \gamma^{m} d^{2}\right)\left(\bar{\lambda} r d^{1} d^{1}\right)\left(\bar{\lambda} r d^{1} d^{3}\right)\left(\bar{\lambda} r d^{2} d^{2}\right)\left(\bar{\lambda} r d^{2} d^{3}\right)\left(\bar{\lambda} r d^{3} d^{3}\right) \\
& +\left(\bar{\lambda} \gamma^{m} d^{3}\right)\left(\bar{\lambda} r d^{1} d^{1}\right)\left(\bar{\lambda} r d^{1} d^{2}\right)\left(\bar{\lambda} r d^{2} d^{2}\right)\left(\bar{\lambda} r d^{2} d^{3}\right)\left(\bar{\lambda} r d^{3} d^{3}\right)
\end{aligned}
$$

while $B_{(4,3,4)}^{m}$ and $B_{(4,4,3)}^{m}$ are obtained from $B_{(3,4,4)}^{m}$ by swapping $d_{\alpha}^{1} \leftrightarrow d_{\alpha}^{2}$ and $d_{\alpha}^{1} \leftrightarrow d_{\alpha}^{3}$, respectively. Furthermore, note that $B_{(3,4,4)}^{m}$ is symmetric under $d_{\alpha}^{2} \leftrightarrow d_{\alpha}^{3}$.

Taking into account that $c_{b_{2}}=48\left(\alpha^{\prime} / 2\right)^{-1} c_{b_{1}}$ and using the definition (3.5) leads to the following expression for $A_{11}$,

$$
\begin{aligned}
A_{11}= & \frac{\sqrt{2} 2^{-93} \kappa^{4} e^{4 \lambda}}{\pi^{42} 3^{10}(11 ! 5 !)^{6}}\left(\frac{\alpha^{\prime}}{2}\right)^{22} \int \frac{d^{2} \Omega_{I J}}{Z_{3}^{22}} \int_{\Sigma_{4}}\left\langle\prod_{j=1}^{4} e^{i k^{j} \cdot x^{j}}\right\rangle \\
& \times\left|\left\langle\left(\Pi_{m}^{1} D_{(14,15,15)}^{m}+\Pi_{m}^{2} D_{(15,14,15)}^{m}+\Pi_{m}^{3} D_{(15,15,14)}^{m}\right) U^{1} U^{2} U^{3} U^{4}\right\rangle_{(-8)}\right|^{2} .
\end{aligned}
$$

Each external vertex $U^{i}$ contribute through the term $\left(\alpha^{\prime} / 2\right)\left(d W^{i}\right)\left(z_{i}\right)$ and the integration over the $d$ zero-modes can be carried out by using the following formulae,

$$
\begin{aligned}
& D_{(14,15,15)}^{m}\left(d^{1} W^{1}\right)\left(d^{1} W^{2}\right)\left(d^{2} W^{3}\right)\left(d^{3} W^{4}\right)=+(11 ! 5 !)^{3} 96^{2} c_{d}^{3} S_{1234}^{m}(\lambda, \bar{\lambda}, r) \\
& D_{(15,14,15)}^{m}\left(d^{2} W^{1}\right)\left(d^{2} W^{2}\right)\left(d^{1} W^{3}\right)\left(d^{3} W^{4}\right)=-(11 ! 5 !)^{3} 96^{2} c_{d}^{3} S_{1234}^{m}(\lambda, \bar{\lambda}, r) \\
& D_{(15,15,14)}^{m}\left(d^{3} W^{1}\right)\left(d^{3} W^{2}\right)\left(d^{1} W^{3}\right)\left(d^{2} W^{4}\right)=+(11 ! 5 !)^{3} 96^{2} c_{d}^{3} S_{1234}^{m}(\lambda, \bar{\lambda}, r)
\end{aligned}
$$

where

$$
S_{1234}^{m}(\lambda, \bar{\lambda}, r)=S_{1234}^{(1) m}(\lambda, \bar{\lambda}, r)+S_{1234}^{(2) m}(\lambda, \bar{\lambda}, r)-S_{1243}^{(2) m}(\lambda, \bar{\lambda}, r)
$$

and

$$
\begin{aligned}
& S_{1234}^{(1) m}(\lambda, \bar{\lambda}, r)=2\left(\bar{\lambda} \gamma^{m} \gamma^{a_{1}} \lambda\right)\left(\bar{\lambda} \gamma_{m_{1} n_{1} p_{1}} r\right)\left(\bar{\lambda} \gamma_{m_{2} n_{2} p_{2}} r\right)\left(\bar{\lambda} \gamma_{m_{3} n_{3} p_{3}} r\right)\left(\bar{\lambda} \gamma_{m_{4} n_{4} p_{4}} r\right)\left(\bar{\lambda} \gamma_{m_{5} n_{5} p_{5}} r\right) \\
& \times\left(\lambda \gamma^{a_{2} m_{1} n_{1} p_{1} m_{3}} \lambda\right)\left(\lambda \gamma^{a_{3} m_{2} n_{2} p_{2} m_{5}} \lambda\right)\left(\lambda \gamma^{n_{3} m_{4} n_{4} p_{4} n_{5}} \lambda\right) \\
& \times\left(W^{1} \gamma^{a_{1} a_{2} a_{3}} W^{2}\right)\left(\lambda \gamma^{p_{3}} W^{3}\right)\left(\lambda \gamma^{p_{5}} W^{4}\right) \\
& S_{1234}^{(2) m}(\lambda, \bar{\lambda}, r)=96\left(\bar{\lambda} \gamma^{m} \gamma^{m_{3}} \lambda\right)\left(\bar{\lambda} \gamma_{m_{1} n_{1} p_{1}} r\right)\left(\bar{\lambda} \gamma_{m_{2} n_{2} p_{2}} r\right)\left(\bar{\lambda} \gamma_{m_{3} n_{3} p_{3}} r\right)\left(\bar{\lambda} \gamma_{m_{4} n_{4} p_{4}} r\right)\left(\bar{\lambda} \gamma_{m_{5} n_{5} p_{5}} r\right) \\
& \times\left(\lambda \gamma^{m_{1} m_{2} n_{2} p_{2} m_{5}} \lambda\right)\left(\lambda \gamma^{n_{3} m_{4} n_{4} p_{4} n_{5}} \lambda\right) \\
& \times\left(\lambda \gamma^{n_{1}} W^{1}\right)\left(\lambda \gamma^{p_{1}} W^{2}\right)\left(\lambda \gamma^{p_{3}} W^{3}\right)\left(\lambda \gamma^{p_{5}} W^{4}\right) .
\end{aligned}
$$

Note from the definition (3.19) that $S_{1234}^{m}(\lambda, \bar{\lambda}, r)$ is symmetric in the particle labels (12) and antisymmetric in [34]. The explicit expression for $S_{1234}^{(1)}(\lambda, \bar{\lambda}, r)$ is symmetric in (12) and 
antisymmetric in [34] whereas $S_{1234}^{(2)}(\lambda, \bar{\lambda}, r)$ is symmetric in (12), so (3.20) indeed has the required symmetries. According to the procedure of Appendix A we write $S_{1234}^{m}(\lambda, \bar{\lambda}, r)=$ $(\lambda \bar{\lambda})^{6} S_{1234}^{m}(\lambda, r)$ and drop the arguments $(\lambda, r)$ in the following.

After expanding $d_{\alpha}(z)=\hat{d}_{\alpha}(z)+d_{\alpha}^{I} w_{I}(z)$ and using (3.19) together with the symmetry properties of $S_{1234}^{m}$ it is a matter of bookkeeping the permutations to arrive at

$$
\left(\Pi_{m}^{1} D_{(14,15,15)}^{m}+\Pi_{m}^{2} D_{(15,14,15)}^{m}+\Pi_{m}^{3} D_{(15,15,14)}^{m}\right) U^{1} U^{2} U^{3} U^{4}=\left(\frac{\alpha^{\prime}}{2}\right)^{4}(11 ! 5 !)^{3} 96^{2} c_{d}^{3}(\lambda \bar{\lambda})^{6} \mathcal{K}_{11}
$$

where

$$
\begin{aligned}
\mathcal{K}_{11}= & +S_{1234}^{m} \Delta^{m}\left(z_{1}, z_{2} ; z_{3} ; z_{4}\right)+S_{1324}^{m} \Delta^{m}\left(z_{1}, z_{3} ; z_{2} ; z_{4}\right)+S_{1423}^{m} \Delta^{m}\left(z_{1}, z_{4} ; z_{2} ; z_{3}\right) \\
& +S_{2314}^{m} \Delta^{m}\left(z_{2}, z_{3} ; z_{1} ; z_{4}\right)+S_{2413}^{m} \Delta^{m}\left(z_{2}, z_{4} ; z_{1} ; z_{3}\right)+S_{3412}^{m} \Delta^{m}\left(z_{3}, z_{4} ; z_{1} ; z_{2}\right) .
\end{aligned}
$$

Finally,

$$
A_{11}=\kappa^{4} e^{4 \lambda} \frac{\sqrt{2} \pi^{6}}{2^{25} 3^{6}}\left(\frac{\alpha^{\prime}}{2}\right)^{6} \int \frac{d^{2} \Omega_{I J}}{Z_{3}^{-10}} \int_{\Sigma_{4}}\left\langle\left|\mathcal{K}_{11}\right|^{2}\right\rangle_{(-2)}\left\langle\prod_{j=1}^{4} e^{i k^{j} \cdot x^{j}}\right\rangle .
$$

Therefore from (3.11) and (3.24) the three-loop amplitude $\mathcal{A}_{3}=A_{12}+A_{11}$ becomes

$$
\mathcal{A}_{3}=\kappa^{4} e^{4 \lambda} \frac{\sqrt{2} \pi^{6}}{2^{23} 3^{6}}\left(\frac{\alpha^{\prime}}{2}\right)^{6} \int_{\mathcal{M}_{3}} \frac{d^{2} \Omega_{I J}}{(\operatorname{det}(2 \operatorname{Im} \Omega))^{5}} \int_{\Sigma_{4}}\left[\left\langle|\mathcal{F}|^{2}\right\rangle_{(-3)}+\left\langle|\mathcal{T}|^{2}\right\rangle_{(-3)}\right]\left\langle\prod_{j=1}^{4} e^{i k^{j} \cdot x^{j}}\right\rangle
$$

where (2.16) has been used,

$$
\begin{aligned}
\mathcal{F}= & +M_{12,3,4} \Delta\left(z_{2} ; z_{3} ; z_{4}\right) X_{12}+M_{13,2,4} \Delta\left(z_{3} ; z_{2} ; z_{4}\right) X_{13}+M_{14,2,3} \Delta\left(z_{4} ; z_{2} ; z_{3}\right) X_{14} \\
& +M_{23,1,4} \Delta\left(z_{3} ; z_{1} ; z_{4}\right) X_{23}+M_{24,1,3} \Delta\left(z_{4} ; z_{1} ; z_{3}\right) X_{24}+M_{34,1,2} \Delta\left(z_{4} ; z_{1} ; z_{2}\right) X_{34} \\
\mathcal{T}= & +T_{1234}^{m} \Delta^{m}\left(z_{1}, z_{2} ; z_{3} ; z_{4}\right)+T_{1324}^{m} \Delta^{m}\left(z_{1}, z_{3} ; z_{2} ; z_{4}\right)+T_{1423}^{m} \Delta^{m}\left(z_{1}, z_{4} ; z_{2} ; z_{3}\right) \\
& +T_{2314}^{m} \Delta^{m}\left(z_{2}, z_{3} ; z_{1} ; z_{4}\right)+T_{2413}^{m} \Delta^{m}\left(z_{2}, z_{4} ; z_{1} ; z_{3}\right)+T_{3412}^{m} \Delta^{m}\left(z_{3}, z_{4} ; z_{1} ; z_{2}\right)
\end{aligned}
$$

and (the other $T_{i j k l}^{m}$ follow from relabeling),

$$
T_{1234}^{m}=L_{1342}^{m}+L_{2341}^{m}+\frac{5}{2} S_{1234}^{m} .
$$

The factor 5 in $(3.27)$ is due to $\langle\ldots\rangle_{(-2)}=5\langle\ldots\rangle_{(-3)}$ and follows from $(2.21)$. The factor $1 / 2$ accounts for the different overall normalizations of (3.24) and (3.11).

After using (2.41) the three-loop amplitude (3.25) becomes

$$
\mathcal{A}_{3}=(2 \pi)^{10} \delta^{(10)}(k) \frac{\kappa^{4} e^{4 \lambda}}{2^{31} 3^{8} 5^{2} 7^{2}}\left(\frac{\alpha^{\prime}}{2}\right)^{5} \int_{\mathcal{M}_{3}} \frac{d^{2} \Omega_{I J}}{(\operatorname{det}(2 \operatorname{Im} \Omega))^{5}} \int_{\Sigma_{4}}\left[\left\langle|\mathcal{F}|^{2}\right\rangle+\left\langle|\mathcal{T}|^{2}\right\rangle\right] \mathcal{I}\left(s_{i j}\right) \text {. }
$$




\subsection{The low-energy limit $D^{6} R^{4}$}

Since the superfields in $\mathcal{F}$ and $\mathcal{T}$ have component expansions terms of order $k^{4} F_{m n}^{4}$ and $k^{3} F_{m n}^{4}$ one might naively expect that only $\left\langle|\mathcal{T}|^{2}\right\rangle$ in $(3.25)$ contributes to the low-energy limit of order $D^{6} R^{4}$. However some integrals in $\left\langle|\mathcal{F}|^{2}\right\rangle$ contain kinematic poles which reduce its contribution from $D^{8} R^{4}$ to $D^{6} R^{4}$. In fact, the integration by parts identities of Appendix B show that ${ }^{5}$

$$
\begin{aligned}
\int_{\Sigma_{4}}\left\langle|\mathcal{F}|^{2}\right\rangle \mathcal{I}\left(s_{i j}\right) & =-\pi\left(\frac{\alpha^{\prime}}{2}\right)\langle\mathcal{K}\rangle \int_{\Sigma_{4}} \Omega_{12} \Delta\left(z_{2} ; z_{3} ; z_{4}\right) \bar{\Delta}\left(z_{1} ; z_{3} ; z_{4}\right)+\mathcal{O}\left(\alpha^{\prime 2}\right) \\
& =-12 \pi\left(\frac{\alpha^{\prime}}{2}\right)\langle\mathcal{K}\rangle \operatorname{det}(2 \operatorname{Im} \Omega)+\mathcal{O}\left(\alpha^{\prime 2}\right)
\end{aligned}
$$

where

$$
\mathcal{K}=\frac{\left|T_{23,1,4}\right|^{2}}{s_{23}}+\frac{\left|T_{24,1,3}\right|^{2}}{s_{24}}+\frac{\left|T_{34,1,2}\right|^{2}}{s_{34}}+\frac{\left|T_{12,3,4}\right|^{2}}{s_{12}}+\frac{\left|T_{13,2,4}\right|^{2}}{s_{13}}+\frac{\left|T_{14,2,3}\right|^{2}}{s_{14}}
$$

is such that $\langle\mathcal{K}\rangle$ is also of order $D^{6} R^{4}$.

To compute the low-energy limit of $\int_{\Sigma_{4}}|\mathcal{T}|^{2} \mathcal{I}\left(s_{i j}\right)$ it will be convenient to use the symmetry relations ${ }^{6}$

$$
T_{1234}^{m}+T_{3124}^{m}+T_{2314}^{m}=0, \quad T_{1234}^{m}=T_{2134}^{m}, \quad T_{1234}^{m}=-T_{1243}^{m}
$$

to eliminate $T_{1234}^{m}, T_{1324}^{m}$ and $T_{2314}^{m}$ from (3.26) (using, for instance, $T_{1234}^{m}=T_{1423}^{m}+T_{2413}^{m}$ ). Doing this and applying (2.31) one arrives at

$$
\mathcal{T}=T_{1423}^{m} \sum_{I=1}^{3} \Pi_{I}^{m} w_{I}\left(z_{1}\right) \Delta_{234}+T_{2413}^{m} \sum_{I=1}^{3} \Pi_{I}^{m} w_{I}\left(z_{2}\right) \Delta_{134}+T_{3412}^{m} \sum_{I=1}^{3} \Pi_{I}^{m} w_{I}\left(z_{3}\right) \Delta_{124}
$$

5 In the first version of this paper the result of the integral $\int_{\Sigma_{4}} \Omega_{12} \Delta\left(z_{2} ; z_{3} ; z_{4}\right) \bar{\Delta}\left(z_{1} ; z_{3} ; z_{4}\right)$ was incorrectly stated as $36 \operatorname{det}(2 \operatorname{Im} \Omega)$ instead of $12 \operatorname{det}(2 \operatorname{Im} \Omega)$. Using a notation where $X_{I J} \equiv$ $\operatorname{Im} \Omega_{I J}$, the definitions (2.6) and (2.30) imply that the integral is

$$
\begin{aligned}
& =\int_{\Sigma_{4}} w_{I}\left(z_{1}\right) X_{I J}^{-1} \bar{w}_{J}\left(\bar{z}_{2}\right) \epsilon^{K L M} w_{K}\left(z_{2}\right) w_{L}\left(z_{3}\right) w_{M}\left(z_{4}\right) \epsilon^{P Q R} \bar{w}_{P}\left(\bar{z}_{1}\right) \bar{w}_{Q}\left(\bar{z}_{3}\right) \bar{w}_{R}\left(\bar{z}_{4}\right) \\
& =2^{4} X_{I P} X_{I J}^{-1} X_{J K} \epsilon^{K L M} \epsilon^{P Q R} X_{L Q} X_{M R}=2^{4} X_{P K} X_{Q L} X_{R M} \epsilon^{K L M} \epsilon^{P Q R}=12 \operatorname{det}(2 \operatorname{Im} \Omega)
\end{aligned}
$$

where $X_{I J}^{-1} X_{J K}=\delta_{I K}, X_{P K} X_{Q L} X_{R M} \epsilon^{K L M} \epsilon^{P Q R}=3 ! \operatorname{det}(X)$ and $\operatorname{det}(2 X)=2^{3} \operatorname{det}(X)$ have been used. A similar mistake in the calculation of $\int_{\Sigma_{4}}|\mathcal{T}|^{2}$ led to an incorrect overall coefficient for the low-energy limit which was bigger by a factor of 3 .

6 We thank Piotr Tourkine for pointing out the first symmetry relation in (3.31) and Oliver Schlotterer for emphasizing its role in simplifying the expression of $\mathcal{T}$ and $\mathcal{L} \cdot \tilde{\mathcal{L}}$. 
After setting $\mathcal{I}\left(s_{i j}\right)=1$ (since there are no kinematic poles), the contribution of $\int_{\Sigma_{4}}\left\langle|T|^{2}\right\rangle$ to the low-energy limit require the following integrals

$$
\begin{aligned}
& \int_{\Sigma_{4}} \Pi_{I}^{m} \bar{\Pi}_{J}^{n} w_{I}\left(z_{1}\right) \bar{w}_{J}\left(\bar{z}_{1}\right) \Delta_{234} \bar{\Delta}_{234}=-36 \pi \eta^{m n}\left(\frac{\alpha^{\prime}}{2}\right) \operatorname{det}(2 \operatorname{Im} \Omega) \\
& \int_{\Sigma_{4}} \Pi_{I}^{m} \bar{\Pi}_{J}^{n} w_{I}\left(z_{1}\right) \bar{w}_{J}\left(\bar{z}_{2}\right) \Delta_{234} \bar{\Delta}_{341}=-12 \pi \eta^{m n}\left(\frac{\alpha^{\prime}}{2}\right) \operatorname{det}(2 \operatorname{Im} \Omega) .
\end{aligned}
$$

and yields

$$
\int_{\Sigma_{4}}|\mathcal{T}|^{2}=-12 \pi\left(\frac{\alpha^{\prime}}{2}\right) \operatorname{det}(2 \operatorname{Im} \Omega) \mathcal{L} \cdot \tilde{\mathcal{L}}
$$

where

$$
\mathcal{L} \cdot \tilde{\mathcal{L}} \equiv\left|T_{1234}^{m}\right|^{2}+\left|T_{1324}^{m}\right|^{2}+\left|T_{1423}^{m}\right|^{2}+\left|T_{2314}^{m}\right|^{2}+\left|T_{2413}^{m}\right|^{2}+\left|T_{3412}^{m}\right|^{2}
$$

Therefore the low-energy limit of the three-loop amplitude (3.28) is given by

$$
\begin{aligned}
\mathcal{A}_{3} & =-(2 \pi)^{10} \delta^{(10)}(k)\left(\frac{\alpha^{\prime}}{2}\right)^{6}\langle\mathcal{K}+\mathcal{L} \cdot \tilde{\mathcal{L}}\rangle \kappa^{4} e^{4 \lambda} \frac{\pi}{2^{29} 3^{7} 5^{2} 7^{2}} \int_{\mathcal{M}_{3}} \frac{d^{2} \Omega_{I J}}{(\operatorname{det}(2 \operatorname{Im} \Omega))^{4}} \\
& =-(2 \pi)^{10} \delta^{(10)}(k)\left(\frac{\alpha^{\prime}}{2}\right)^{6}\langle\mathcal{K}+\mathcal{L} \cdot \tilde{\mathcal{L}}\rangle \kappa^{4} e^{4 \lambda} \frac{\pi \zeta_{6}}{2^{35} 3^{10} 5^{3} 7^{2}}
\end{aligned}
$$

where the integral is $2^{-12} \mathrm{Vol}_{3}$ and we used $\zeta_{6}=\pi^{6} / 945$. A long calculation gives [25]

$$
\langle\mathcal{K}+\mathcal{L} \cdot \tilde{\mathcal{L}}\rangle=-2^{35} 3^{7} 5^{3} 7^{2}\left(s_{12}^{3}+s_{13}^{3}+s_{14}^{3}\right) K \bar{K}
$$

irrespective of whether it is for type IIA or IIB, confirming the theorem of [7]. Therefore

$$
\mathcal{A}_{3}=(2 \pi)^{10} \delta^{(10)}(k) \kappa^{4} e^{4 \lambda} \frac{\pi \zeta_{6}}{3^{3}}\left(\frac{\alpha^{\prime}}{2}\right)^{6}\left(s_{12}^{3}+s_{13}^{3}+s_{14}^{3}\right) K \bar{K}
$$

is the low-energy limit of the type IIA and IIB three-loop amplitude.

\section{Perturbative calculations versus S-duality predictions}

We first review the one- and two-loop comparisons between S-duality predictions and perturbative amplitude calculations of $[21,19]$ using our conventions. After that we extend their analysis to include the three-loop result (3.38). We will find that the amplitude we computed in (3.38) agrees with the prediction of Green and Vanhove [12]. 


\subsection{One- and two-loops}

The closed-string massless four-point amplitudes at genus 0,1 and 2 computed in [11] (including their overall coefficients) are given by (see also [19]),

$$
\begin{aligned}
& \mathcal{A}_{0}=(2 \pi)^{10} \delta^{(10)}(k)\left(\frac{\alpha^{\prime}}{2}\right)^{3} K \bar{K} \kappa^{4} e^{-2 \lambda} \frac{\sqrt{2}}{2^{16} \pi^{5}} \mathcal{B}_{0}\left(s_{i j}\right) \\
& \mathcal{A}_{1}=(2 \pi)^{10} \delta^{(10)}(k)\left(\frac{\alpha^{\prime}}{2}\right)^{3} K \bar{K} \kappa^{4} \frac{1}{2^{14} \pi^{2}} \int_{\mathcal{M}_{1}} \frac{d^{2} \tau}{\tau_{2}^{2}} \mathcal{B}_{1}\left(s_{i j} \mid \tau\right) \\
& \mathcal{A}_{2}=(2 \pi)^{10} \delta^{(10)}(k)\left(\frac{\alpha^{\prime}}{2}\right)^{3} K \bar{K} \kappa^{4} e^{2 \lambda} \frac{\sqrt{2}}{2^{15}} \int_{\mathcal{M}_{2}} \frac{d^{2} \Omega_{I J}}{(\operatorname{det}(\operatorname{Im} \Omega))^{3}} \mathcal{B}_{2}\left(s_{i j} \mid \Omega\right)
\end{aligned}
$$

where $^{7}[21,19]$,

$$
\begin{aligned}
\mathcal{B}_{0}\left(s_{i j}\right) & =\frac{\Gamma\left(-\alpha^{\prime} s_{12} / 2\right) \Gamma\left(-\alpha^{\prime} s_{13} / 2\right) \Gamma\left(-\alpha^{\prime} s_{14} / 2\right)}{\Gamma\left(1+\alpha^{\prime} s_{12} / 2\right) \Gamma\left(1+\alpha^{\prime} s_{13} / 2\right) \Gamma\left(1+\alpha^{\prime} s_{14} / 2\right)} \\
& =\frac{3}{\sigma_{3}}+2 \zeta_{3}+\zeta_{5} \sigma_{2}+\frac{2}{3} \zeta_{3}^{2} \sigma_{3}+\cdots \\
\mathcal{B}_{1}\left(s_{i j} \mid \tau\right) & =\int \prod_{i=2}^{4} \frac{d^{2} z_{i}}{\tau_{2}} \mathcal{I}\left(s_{i j}\right)=2^{3}\left(1+\frac{\zeta_{3}}{3} \sigma_{3}+\cdots\right. \\
\mathcal{B}_{2}\left(s_{i j} \mid \Omega\right) & =\int_{\Sigma^{4}} \frac{|\mathcal{Y}|^{2}}{(\operatorname{det}(\operatorname{Im} \Omega))^{2}} \mathcal{I}\left(s_{i j}\right)=2^{7} \sigma_{2}+\cdots
\end{aligned}
$$

Plugging in the volume of the moduli spaces (2.36) one obtains the following low-energy expansions,

$$
\begin{aligned}
& \mathcal{A}_{0}=(2 \pi)^{10} \delta^{(10)}(k)\left(\frac{\alpha^{\prime}}{2}\right)^{3} K \bar{K} \kappa^{4} e^{-2 \lambda} \frac{\sqrt{2}}{2^{16} \pi^{5}}\left[\frac{3}{\sigma_{3}}+2 \zeta_{3}+\zeta_{5} \sigma_{2}+\frac{2}{3} \zeta_{3}^{2} \sigma_{3}+\cdots\right. \\
& \mathcal{A}_{1}=(2 \pi)^{10} \delta^{(10)}(k)\left(\frac{\alpha^{\prime}}{2}\right)^{3} K \bar{K} \kappa^{4} \frac{1}{2^{10} 3 \pi}\left[1+\frac{\zeta_{3}}{3} \sigma_{3}+\cdots\right. \\
& \mathcal{A}_{2}=(2 \pi)^{10} \delta^{(10)}(k)\left(\frac{\alpha^{\prime}}{2}\right)^{3} K \bar{K} \kappa^{4} e^{2 \lambda} \frac{\sqrt{2} \pi^{3}}{2^{6} 3^{3} 5}\left[\sigma_{2}+\cdots\right.
\end{aligned}
$$

The $S L(2, \mathbb{Z})$-duality predictions for the perturbative effective action are [32,33,12]

$$
\begin{aligned}
& S^{\alpha^{\prime 3}}=C_{1} \int d^{10} x \sqrt{-g} \mathcal{R}^{4}\left(2 \zeta_{3} e^{-2 \phi}+\frac{2 \pi^{2}}{3}\right) \\
& S^{\alpha^{\prime 5}}=C_{2} \int d^{10} x \sqrt{-g} D^{4} \mathcal{R}^{4}\left(2 \zeta_{5} e^{-2 \phi}+\frac{8}{3} \zeta_{4} e^{2 \phi}\right) \\
& S^{\alpha^{\prime 6}}=C_{3} \int d^{10} x \sqrt{-g} D^{6} \mathcal{R}^{4}\left(4 \zeta_{3}^{2} e^{-2 \phi}+8 \zeta_{2} \zeta_{3}+\frac{48}{5} \zeta_{2}^{2} e^{2 \phi}+\frac{8}{9} \zeta_{6} e^{4 \phi}\right) .
\end{aligned}
$$

$7 \mathcal{B}_{0}\left(s_{i j}\right)$ here is $\frac{1}{2 \pi} C(s, t, u)$ from [11]. Furthermore, see [30] for a recent attempt to evaluate non-leading terms at two-loops and [31] for an elegant way to rewrite the tree-level expansion. 
where the precise definitions of $R^{4}, D^{4} \mathcal{R}^{4}$ and $D^{6} \mathcal{R}^{4}$ and the constants $C_{\{1,2,3\}}$ will not be needed in the following discussion since only the ratios of the interactions at different loop orders will be important.

Matching the ratios of the $\alpha^{\prime 3}$ interactions at one-loop and tree-level leads to a relation between $e^{\phi}$ and $e^{\lambda}$,

$$
\frac{\sqrt{2} 2^{4} \pi^{4} e^{2 \lambda}}{3 \zeta_{3}}=\frac{e^{2 \phi} \pi^{2}}{3 \zeta_{3}} \rightarrow e^{2 \phi}=e^{2 \lambda} \sqrt{2} 2^{4} \pi^{2}
$$

where the left-hand side follows from the amplitudes while the right-hand side from the effective action (4.6). Now one can compare the S-duality predictions for the amplitudes at order $\alpha^{\prime 5}$ and $\alpha^{\prime 6}$ (denoted with a Latin capital $A^{\alpha^{\prime n}}$ ) and the perturbative results.

For the $\alpha^{\prime 5}$ interaction, the ratio between the two-loop and tree-level interactions in the effective action $(4.7)$ is $\frac{4 \zeta_{4}}{3 \zeta_{5}} e^{4 \phi}$ and leads to the prediction

$$
A_{2}^{\alpha^{\prime 5}}=\mathcal{A}_{0}^{\alpha^{\prime 5}} \frac{2^{11} \pi^{4} \zeta_{4}}{3 \zeta_{5}} e^{4 \lambda}=(2 \pi)^{10} \delta^{(10)}(k) \kappa^{4} e^{2 \lambda}\left(\frac{\alpha^{\prime}}{2}\right)^{3} K \bar{K} \frac{\sqrt{2} \zeta_{4}}{2^{5} 3 \pi} \sigma_{2}
$$

which agrees with the two-loop perturbative calculation (4.5) (recall that $\zeta_{4}=\pi^{4} / 90$ ).

For the $\alpha^{\prime 6}$ interaction, the ratio between the one-loop and tree-level terms following from the effective action (4.8) is $2 \zeta_{2} / \zeta_{3} e^{2 \phi}=\zeta_{2} / \zeta_{3} \sqrt{2} 2^{5} \pi^{2} e^{2 \lambda}$ and implies

$$
A_{1}^{\alpha^{\prime 6}}=(2 \pi)^{10} \delta^{(10)}(k)\left(\frac{\alpha^{\prime}}{2}\right)^{3} K \bar{K} \kappa^{4} \frac{\zeta_{2} \zeta_{3}}{2^{9} 3 \pi^{3}} \sigma_{3},
$$

which agrees with the one-loop perturbative calculation (4.4), in accord with the analysis of $[21]$.

\subsection{Three-loops}

Similarly, the ratio between the three-loop and tree-level terms of (4.8)

$$
\frac{2 \zeta_{6}}{9 \zeta_{3}^{2}} e^{6 \phi}=\frac{\sqrt{2} 2^{14} \pi^{6} \zeta_{6}}{9 \zeta_{3}^{2}} e^{6 \lambda}=\frac{\mathcal{A}_{3}^{\alpha^{\prime 6}}}{\mathcal{A}_{0}^{\alpha^{\prime 6}}}
$$

predicts the following three-loop amplitude

$$
A_{3}^{\alpha^{\prime 6}}=(2 \pi)^{10} \delta^{(10)}(k)\left(\frac{\alpha^{\prime}}{2}\right)^{3} K \bar{K} \kappa^{4} e^{4 \lambda} \frac{\pi \zeta_{6}}{3^{3}} \sigma_{3}
$$

in complete agreement with the first principles perturbative calculation (3.38). 
Acknowledgements: CRM thanks Michael Green for many discussions over the years and for his continuous interest. We also thank Edward Witten for making it clear that no symmetry argument could account for an overall factor of $1 / 3$ as argued in the first version of this paper. CRM also thanks Oliver Schlotterer for collaboration on related topics. We would like to thank Nathan Berkovits for discussions and to Oscar A. Bedoya for reading the manuscript. H.G thanks Freddy Cachazo for his comments and discussions about Riemann surfaces and Andrei Mikhailov for discussions about the pure spinor formalism. H.G is grateful to the Albert-Einstein-Institut, DAMTP, IFT-UNESP and especially to the Perimeter Institute for Theoretical Physics for warm hospitality during stages of this work. CRM thanks the Albert-Einstein-Institut in Potsdam for the wonderful work environment during the early stages of this work. The work of H.G is supported by FAPESP grant 2011/13013-8, 13/11409-7 and CRM acknowledges support by the European Research Council Advanced Grant No. 247252 of Michael Green.

\section{Appendix A. A general formula for integration of pure spinors}

Component expansions of a general ghost-number-three pure spinor superspace expression of the form $\lambda^{\alpha_{1}} \ldots \lambda^{\alpha_{n+3}} \bar{\lambda}_{\beta_{1}} \ldots \bar{\lambda}_{\beta_{n}} f_{\alpha_{1} \ldots \alpha_{n+3}}^{\beta_{1} \ldots \beta_{n}}(\theta, r)$ can be computed most conveniently by first rewriting it in the form $(\lambda \bar{\lambda})^{n} \lambda^{\alpha_{1}} \ldots \lambda^{\alpha_{3}} f_{\alpha_{1} \ldots \alpha_{3}}(\theta, r)$. Doing that allows the straightforward application of the formula (2.21) and the identities listed in the appendix of [34]. The case $n=1$ was discussed in [35], now the solution for general $n$ will be presented.

Let $\mathcal{T}_{\beta_{1} \ldots \beta_{n}}^{\alpha_{1} \ldots \alpha_{n}}$ denote a $S O(10)$-invariant tensor which is symmetric and $\gamma$-traceless in both sets of indices. When $n$ is even there is $(n / 2+1)$-dimensional basis [36],

$$
\begin{gathered}
\mathcal{T}_{\beta_{1} \ldots \beta_{n}}^{\alpha_{1} \ldots \alpha_{n}}=\sum_{k=0}^{n / 2} c_{k}^{(n)} T_{k}^{(n)}, \\
T_{k}^{(n)}=\delta_{\left(\beta_{1}\right.}^{\left(\alpha_{1}\right.} \cdots \delta_{\beta_{n-2 k}}^{\alpha_{n-2 k}}(\gamma \cdot \gamma)_{\beta_{n-2 k+1} \beta_{n-2 k+2}}^{\alpha_{n-2 k+1} \alpha_{n-2 k+2}} \cdots(\gamma \cdot \gamma)_{\left.\beta_{n-1} \beta_{n}\right)}^{\left.\alpha_{n-1} \alpha_{n}\right)},
\end{gathered}
$$

and $(\gamma \cdot \gamma)_{\beta_{1} \beta_{2}}^{\alpha_{1} \alpha_{2}} \equiv \gamma_{m}^{\alpha_{1} \alpha_{2}} \gamma_{\beta_{1} \beta_{2}}^{m}$. Imposing the $\gamma$-traceless condition leads to a recurrence relation for the coefficients $c_{k}^{(n)}$ [37] and the normalization condition $T_{\alpha_{1} \ldots \alpha_{n}}^{\alpha_{1} \ldots \alpha_{n}}=1$ relates the coefficient $c_{0}^{(n)}$ with the dimension of the pure spinor representation $N_{n} \equiv \operatorname{dim}([0000 n])$,

$$
c_{k+1}^{(n)}=-\frac{(n-2 k)(n-2 k-1)}{8(k+1)(n-k+2)} c_{k}^{(n)}, \quad c_{0}^{(n)}=1 / N_{n},
$$


where $^{8}$

$$
\begin{aligned}
N_{n} & =\frac{1}{302400}(n+7)(n+6)(n+5)^{2}(n+4)^{2}(n+3)^{2}(n+2)(n+1) \\
& =16,126,672,2772,9504,28314 \ldots
\end{aligned}
$$

When $m=n-1$ is odd, the tensor $\mathcal{T}_{\beta_{1} \ldots \beta_{m}}^{\alpha_{1} \ldots \alpha_{m}}$ can be obtained from (A.1) by contracting a pair of indices; $\mathcal{T}_{\beta_{1} \ldots \beta_{m}}^{\alpha_{1} \ldots \alpha_{m}}=\mathcal{T}_{\beta_{1} \ldots \beta_{m} \alpha_{m+1}}^{\alpha_{1} \ldots \alpha_{m} \alpha_{m+1}}$.

The explicit expressions for the first few tensors read as follows,

$$
\begin{aligned}
& \mathcal{T}_{\beta_{1}}^{\alpha_{1}}=\frac{1}{16} \delta_{\beta_{1}}^{\alpha_{1}} \\
& \mathcal{T}_{\beta_{1} \beta_{2}}^{\alpha_{1} \alpha_{2}}=\frac{1}{126}\left[\delta_{\beta_{1}}^{\left(\alpha_{1}\right.} \delta_{\beta_{2}}^{\left.\alpha_{2}\right)}-\frac{1}{16}(\gamma \cdot \gamma)_{\beta_{1} \beta_{2}}^{\alpha_{1} \alpha_{2}}\right] \\
& \mathcal{T}_{\beta_{1} \ldots \beta_{3}}^{\alpha_{1} \ldots \alpha_{3}}=\frac{1}{672}\left[\delta_{\beta_{1}}^{\left(\alpha_{1}\right.} \cdots \delta_{\beta_{3}}^{\left.\alpha_{3}\right)}-\frac{3}{20} \delta_{\left(\beta_{1}\right.}^{\left(\alpha_{1}\right.}(\gamma \cdot \gamma)_{\left.\beta_{2} \beta_{3}\right)}^{\left.\alpha_{2} \alpha_{3}\right)}\right] \text {, } \\
& \mathcal{T}_{\beta_{1} \ldots \beta_{4}}^{\alpha_{1} \ldots \alpha_{4}}=\frac{1}{2772}\left[\delta_{\beta_{1}}^{\left(\alpha_{1}\right.} \cdots \delta_{\beta_{4}}^{\left.\alpha_{4}\right)}-\frac{1}{4} \delta_{\left(\beta_{1}\right.}^{\left(\alpha_{1}\right.} \delta_{\beta_{2}}^{\alpha_{2}}(\gamma \cdot \gamma)_{\left.\beta_{3} \beta_{4}\right)}^{\left.\alpha_{3} \alpha_{4}\right)}+\frac{1}{160}(\gamma \cdot \gamma)_{\left(\beta_{1} \beta_{2}\right.}^{\left(\alpha_{1} \alpha_{2}\right.}(\gamma \cdot \gamma)_{\left.\beta_{3} \beta_{4}\right)}^{\left.\alpha_{3} \alpha_{4}\right)}\right] .
\end{aligned}
$$

Using the integration formula of $[10,11]$ and the above $\gamma$-traceless tensors it follows that ${ }^{9}$

$$
\int[d \lambda][d \bar{\lambda}] e^{-(\lambda \bar{\lambda})}(\lambda \bar{\lambda})^{m} \lambda^{\alpha_{1}} \cdots \lambda^{\alpha_{n}} \bar{\lambda}_{\beta_{1}} \cdots \bar{\lambda}_{\beta_{n}}=\left(\frac{A_{g}}{2 \pi}\right)^{11} \frac{12 \Gamma(8+m+n)}{\Gamma(11)} \mathcal{T}_{\beta_{1} \ldots \beta_{n}}^{\alpha_{1} \ldots \alpha_{n}} .
$$

To see this it is enough to check that the right-hand side of (A.5) has the same symmetries of the left-hand side and it is correctly normalized.

Let us define the tensor $\bar{T}^{\alpha \beta \gamma ; \sigma_{1} \ldots \sigma_{5}}$ by [40]

$$
\bar{\lambda}_{\alpha} \bar{\lambda}_{\beta} \bar{\lambda}_{\gamma} \bar{T}^{\alpha \beta \gamma ; \sigma_{1} \ldots \sigma_{5}}=\bar{T}^{\sigma_{1} \ldots \sigma_{5}}
$$

where $\bar{T}^{\sigma_{1} \ldots \sigma_{5}}$ is given in (2.17). Since one can take $\bar{T}^{\alpha \beta \gamma ; \sigma_{1} \ldots \sigma_{5}}$ to be $\gamma$-traceless in the $(\alpha \beta \gamma)$ indices it follows from (A.4) that

$$
\mathcal{T}_{\beta_{1} \beta_{2} \beta_{3}}^{\alpha_{1} \alpha_{2} \alpha_{3}} \bar{T}^{\beta_{1} \beta_{2} \beta_{3} ; \sigma_{1} \ldots \sigma_{5}}=\frac{1}{672} \bar{T}^{\alpha_{1} \alpha_{2} \alpha_{3} ; \sigma_{1} \ldots \sigma_{5}}
$$

Theorem 1. Let $f\left(\lambda^{n+3}, \bar{\lambda}^{n}, \theta\right)$ be a general superfield with ghost-number +3 , then

$$
\left\langle f\left(\lambda^{n+3}, \bar{\lambda}^{n}, \theta\right)\right\rangle_{(m, g)}=\left\langle(\lambda \bar{\lambda})^{n} \hat{f}\left(\lambda^{3}, \theta^{5}\right)\right\rangle_{(m, g)}
$$

$8 N_{n}$ can be obtained from $(1+t)\left(1+4 t+t^{2}\right)(1-t)^{-11}=1+\sum_{n \geq 1} N_{n} t^{n}[38,39]$.

9 Note that all numbers in (2.18) have a geometrical meaning. The number 8 is the ghost anomaly (the first Chern class of the projective pure spinor space), 11 is the complex dimension of the pure spinor space and 12 is the degree of the projective pure spinor space $[10,38]$. 
where

$$
\hat{f}\left(\lambda^{3}, \theta^{5}\right)=672 \lambda^{\beta_{1}} \lambda^{\beta_{2}} \lambda^{\beta_{3}} \mathcal{T}_{\beta_{1} \ldots \beta_{n+3}}^{\sigma_{1} \ldots \sigma_{n+3}} f_{\sigma_{1} \ldots \sigma_{n+3} ; \delta_{1} \ldots \delta_{5}}^{\beta_{4} \ldots \beta_{n+3}} \theta^{\delta_{1}} \ldots \theta^{\delta_{5}}
$$

Proof. Integrating the right-hand side of (A.8) over $[d r]$ and $[d \theta]$ using the measures of (2.14) and the definition (2.20) yields

$$
\begin{array}{r}
\mathrm{RHS}=11 ! 5 ! c_{r} c_{\theta} \int[d \lambda][d \bar{\lambda}] e^{-(\lambda \bar{\lambda})} 672(\lambda \bar{\lambda})^{n+m-3} \lambda^{\beta_{1}} \lambda^{\beta_{2}} \lambda^{\beta_{3}} \bar{\lambda}_{\gamma_{1}} \bar{\lambda}_{\gamma_{2}} \bar{\lambda}_{\gamma_{3}} \\
\times \bar{T}^{\gamma_{1} \gamma_{2} \gamma_{3} ; \delta_{1} \ldots \delta_{5}} \mathcal{T}_{\beta_{1} \ldots \beta_{n+3}}^{\sigma_{1} \ldots \sigma_{n+3}} f_{\sigma_{1} \ldots \sigma_{n+3} ; \delta_{1} \ldots \delta_{5}}^{\beta_{4} \ldots \beta_{n+3}}
\end{array}
$$

Given that the $\mathcal{T}$ tensors are normalized such that $\mathcal{T}_{\beta_{1} \ldots \beta_{p}}^{\alpha_{1} \ldots \alpha_{p}}=1$ the integration over the pure spinors $\lambda$ and $\bar{\lambda}$ using (A.5) leads to

$$
\begin{aligned}
\mathrm{RHS} & =11 ! 5 ! c_{r} c_{\theta}\left(\frac{A_{g}}{2 \pi}\right)^{11} \frac{\Gamma(8+m+n)}{302400} 672 \mathcal{T}_{\gamma_{1} \gamma_{2} \gamma_{3}}^{\beta_{1} \beta_{2} \beta_{3}} \bar{T}^{\gamma_{1} \gamma_{2} \gamma_{3} ; \delta_{1} \ldots \delta_{5}} \mathcal{T}_{\beta_{1} \ldots \beta_{n+3}}^{\sigma_{1} \ldots \sigma_{n+3}} f_{\sigma_{1} \ldots \sigma_{n+3} ; \delta_{1} \ldots \delta_{5}}^{\beta_{4} \ldots \beta_{n+3}} \\
& =11 ! 5 ! c_{r} c_{\theta}\left(\frac{A_{g}}{2 \pi}\right)^{11} \frac{\Gamma(8+m+n)}{302400} \bar{T}^{\beta_{1} \beta_{2} \beta_{3} ; \delta_{1} \ldots \delta_{5}} \mathcal{T}_{\beta_{1} \ldots \beta_{n+3}}^{\sigma_{1} \ldots \sigma_{n+3}} f_{\sigma_{1} \ldots \sigma_{n+3} ; \delta_{1} \ldots \delta_{5}}^{\beta_{4} \ldots \beta_{n+3}} \quad \text { (A.9) }
\end{aligned}
$$

where (A.7) has been used in the second line. However it is easy to show that the evaluation of the left-hand side of (A.8) is equal to (A.9), finishing the proof.

For completeness, note that $\bar{T}^{\alpha \beta \gamma ; \sigma_{1} \ldots \sigma_{5}}$ defined in (A.6) is proportional to the pure spinor correlator $\left\langle\lambda^{\alpha} \lambda^{\beta} \lambda^{\gamma} \theta^{\sigma_{1}} \ldots \theta^{\sigma_{5}}\right\rangle_{(n, g)}$. Indeed, a short computation shows that

$$
\left\langle\lambda^{\alpha} \lambda^{\beta} \lambda^{\gamma} \theta^{\sigma_{1}} \ldots \theta^{\sigma_{5}}\right\rangle_{(n, g)}=\left(\frac{\alpha^{\prime}}{2}\right)^{2}\left(\frac{2 \pi}{A_{g}}\right)^{5 / 2} \frac{\Gamma(8+n)}{302400} \frac{R}{672} \bar{T}^{\alpha \beta \gamma ; \sigma_{1} \ldots \sigma_{5}}
$$

As a consistency check, multiplying both sides by $\gamma_{\alpha \delta_{1}}^{m} \gamma_{\beta \delta_{2}}^{n} \gamma_{\gamma \delta_{3}}^{p}\left(\gamma_{m n p}\right)_{\delta_{4} \delta_{5}}$ recovers (2.21),

$$
\left\langle\left(\lambda \gamma^{m} \theta\right)\left(\lambda \gamma^{n} \theta\right)\left(\lambda \gamma^{p} \theta\right)\left(\theta \gamma_{m n p} \theta\right)\right\rangle_{(n, g)}=\left(\frac{\alpha^{\prime}}{2}\right)^{2}\left(\frac{2 \pi}{A_{g}}\right)^{5 / 2} 2^{7} R \frac{\Gamma(8+n)}{7 !}
$$

where we used that $\bar{T}^{\alpha \beta \gamma ; \delta_{1} \ldots \delta_{5}} \gamma_{\alpha \delta_{1}}^{m} \gamma_{\beta \delta_{2}}^{n} \gamma_{\gamma \delta_{3}}^{p}\left(\gamma_{m n p}\right)_{\delta_{4} \delta_{5}}=5160960$ [41].

As an example, the function $f\left(\lambda^{4}, \bar{\lambda}, \theta\right) \equiv \lambda^{\alpha_{1}} \lambda^{\alpha_{2}} \lambda^{\alpha_{3}} \lambda^{\alpha_{4}} \bar{\lambda}_{\beta_{1}} f_{\alpha_{1} \alpha_{2} \alpha_{3} \alpha_{4}}^{\beta_{1}}(\theta)$ can be easily rewritten according to the Theorem 1 by using

$$
\begin{aligned}
672 \lambda^{\sigma_{1}} \lambda^{\sigma_{2}} \lambda^{\sigma_{3}} \mathcal{T}_{\sigma_{1} \sigma_{2} \sigma_{3} \beta_{1}}^{\alpha_{1} \alpha_{2} \alpha_{3} \alpha_{4}} & =\frac{2}{33}\left[\left\{\delta_{\beta_{1}}^{\alpha_{1}} \lambda^{\alpha_{2}} \lambda^{\alpha_{3}} \lambda^{\alpha_{4}}+\left(\alpha_{1} \leftrightarrow \alpha_{2}, \alpha_{3}, \alpha_{4}\right)\right\}\right. \\
- & \frac{1}{12}\left(\lambda \gamma^{m}\right)_{\beta_{1}}\left\{\gamma_{m}^{\alpha_{1} \alpha_{2}} \lambda^{\alpha_{3}} \lambda^{\alpha_{4}}+\gamma_{m}^{\alpha_{1} \alpha_{3}} \lambda^{\alpha_{2}} \lambda^{\alpha_{4}}+\gamma_{m}^{\alpha_{1} \alpha_{4}} \lambda^{\alpha_{2}} \lambda^{\alpha_{3}}\right. \\
& \left.\left.+\gamma_{m}^{\alpha_{2} \alpha_{3}} \lambda^{\alpha_{1}} \lambda^{\alpha_{4}}+\gamma_{m}^{\alpha_{2} \alpha_{4}} \lambda^{\alpha_{1}} \lambda^{\alpha_{3}}+\gamma_{m}^{\alpha_{3} \alpha_{4}} \lambda^{\alpha_{1}} \lambda^{\alpha_{2}}\right\}\right] .
\end{aligned}
$$




\section{A.1 Factoring $(\lambda \bar{\lambda})^{6}$ from $L_{1234}^{m}(\lambda, \bar{\lambda}, r)$ and $T_{12,3,4}(\lambda, \bar{\lambda}, r)$}

Because of the constraint (2.2) the definition (3.10) can be written as

$$
\begin{aligned}
L_{1234}^{x}(\lambda, \bar{\lambda}, r) & =\left(\bar{\lambda} \gamma^{a} \gamma^{b} \gamma^{c} r\right)\left(\bar{\lambda} \gamma^{\text {def }} r\right)\left(\lambda \gamma^{\text {adefm }} \lambda\right)\left(\bar{\lambda} \gamma^{n} \gamma^{m} \gamma^{p} r\right)\left(\bar{\lambda} \gamma^{q r s} r\right)\left(\lambda \gamma^{n q r s u} \lambda\right) \\
& \times\left(\bar{\lambda} \gamma^{t} \gamma^{u} \gamma^{v} r\right)\left(\bar{\lambda} \gamma^{g h i} r\right)\left(\lambda \gamma^{t g h i b} \lambda\right)\left[\left(\lambda \gamma^{c} W_{1}\right)\left(\lambda \gamma^{p} W_{2}\right)\left(\lambda \gamma^{v} W_{3}\right) A_{4}^{x}\right]
\end{aligned}
$$

Applying the identity $\left(\bar{\lambda} \gamma_{m n p} r\right)\left(\lambda \gamma^{a m n p b} \lambda\right)=48(\lambda \bar{\lambda})\left(\lambda \gamma^{a} \gamma^{b} r\right)-48\left(\lambda \gamma^{a} \gamma^{b} \bar{\lambda}\right)(\lambda r)$ and using the pure spinor constraint gives

$$
\begin{aligned}
L_{1234}^{x}(\lambda, \bar{\lambda}, r)= & -48^{3}(\lambda \bar{\lambda})^{3}\left(\lambda \gamma^{d} \gamma^{a} r\right)\left(\lambda \gamma^{g} \gamma^{e} r\right)\left(\lambda \gamma^{b} \gamma^{i} r\right) \\
& \times\left(\bar{\lambda} \gamma^{a} \gamma^{c} \gamma^{b} r\right)\left(\lambda \gamma^{c} W_{1}\right)\left(\bar{\lambda} \gamma^{e} \gamma^{f} \gamma^{d} r\right)\left(\lambda \gamma^{f} W_{2}\right)\left(\bar{\lambda} \gamma^{i} \gamma^{h} \gamma^{g} r\right)\left(\lambda \gamma^{h} W_{3}\right) A_{4}^{x}
\end{aligned}
$$

where we also renamed indices. Using

$$
\left(\bar{\lambda} \gamma^{m} \gamma^{p} \gamma^{n} r\right)\left(\lambda \gamma^{p} W^{2}\right)=-\left(\lambda \gamma^{p} \gamma^{n} r\right)\left(\bar{\lambda} \gamma^{m} \gamma^{p} W^{2}\right)-\left(\lambda \gamma^{p} \gamma^{m} \bar{\lambda}\right)\left(r \gamma^{n} \gamma^{p} W^{2}\right)
$$

in the last three factors and doing straightforward algebra yields,

$$
\begin{aligned}
L_{1234}^{x}(\lambda, \bar{\lambda}, r)= & 48^{3} 8(\lambda \bar{\lambda})^{5} Q[ \\
& +\left(\lambda \gamma^{a} \gamma^{d} r\right)\left(\lambda \gamma^{c} \gamma^{e} r\right)\left(\lambda \gamma^{b} \gamma^{f} r\right)\left(r \gamma^{a b} W^{1}\right)\left(r \gamma^{d e} W^{2}\right)\left(\bar{\lambda} \gamma^{c f} W^{3}\right) \\
& +\left(\lambda \gamma^{a} \gamma^{d} r\right)\left(\lambda \gamma^{c} \gamma^{e} r\right)\left(\lambda \gamma^{b} \gamma^{f} r\right)\left(r \gamma^{a b} W^{1}\right)\left(\bar{\lambda} \gamma^{d e} W^{2}\right)\left(r \gamma^{c f} W^{3}\right) \\
& +\left(\lambda \gamma^{a} \gamma^{d} r\right)\left(\lambda \gamma^{c} \gamma^{e} r\right)\left(\lambda \gamma^{b} \gamma^{f} r\right)\left(\bar{\lambda} \gamma^{a b} W^{1}\right)\left(r \gamma^{d e} W^{2}\right)\left(r \gamma^{c f} W^{3}\right) \\
& -\left(\lambda \gamma^{c} \gamma^{e} r\right)\left(\lambda \gamma^{b} \gamma^{f} r\right)\left(\lambda \gamma^{a} \gamma^{d} \bar{\lambda}\right)\left(r \gamma^{a b} W^{1}\right)\left(r \gamma^{d e} W^{2}\right)\left(r \gamma^{c f} W^{3}\right) \\
& +\left(\lambda \gamma^{c} \gamma^{e} r\right)\left(\lambda \gamma^{a} \gamma^{d} r\right)\left(\lambda \gamma^{b} \gamma^{f} \bar{\lambda}\right)\left(r \gamma^{a b} W^{1}\right)\left(r \gamma^{d e} W^{2}\right)\left(r \gamma^{c f} W^{3}\right) \\
& \left.+\left(\lambda \gamma^{a} \gamma^{d} r\right)\left(\lambda \gamma^{b} \gamma^{f} r\right)\left(\lambda \gamma^{c} \gamma^{e} \bar{\lambda}\right)\left(r \gamma^{a b} W^{1}\right)\left(r \gamma^{d e} W^{2}\right)\left(r \gamma^{c f} W^{3}\right)\right] \\
+ & 48^{3} 8(\lambda \bar{\lambda})^{6}\left(\lambda \gamma^{a} \gamma^{d} r\right)\left(\lambda \gamma^{c} \gamma^{e} r\right)\left(\lambda \gamma^{b} \gamma^{f} r\right)\left(r \gamma^{a b} W^{1}\right)\left(r \gamma^{d e} W^{2}\right)\left(r \gamma^{c f} W^{3}\right)
\end{aligned}
$$

It is easy to check that (A.15) is totally antisymmetric in [123] as required. The terms proportional to $(\lambda \bar{\lambda})^{5} Q$ will be rewritten using (A.11) and we identified $(\lambda r)=Q$ because of the factor $e^{-(r \theta)}$ in $\mathcal{N}$. Despite the explicit appearance of the BRST charge in some terms, they are not BRST-trivial because of the remaining factor $\bar{\lambda}$. However, since $Q^{2}=0$ and the difference between $\left(\lambda \gamma^{a} \gamma^{d} r\right)$ and $\left(\lambda \gamma^{a d} r\right)$ is proportional to $Q$, one can replace all factors of $\left(\lambda \gamma^{a} \gamma^{d} r\right)$ by $\left(\lambda \gamma^{a d} r\right)$. Doing this replacement is also allowed in the last term because there are no factors of $\bar{\lambda}_{\alpha}$, so the BRST charge vanishes in the cohomology. Similarly, $\left(\lambda \gamma^{a} \gamma^{d} \bar{\lambda}\right)$ 
can be substituted by $\left(\lambda \gamma^{a d} \bar{\lambda}\right)$ since the difference is BRST-trivial due to the resulting factor of $Q(\lambda \bar{\lambda})^{6}$. Therefore (A.15) becomes

$$
L_{1234}^{x}(\lambda, \bar{\lambda}, r)=48^{3} 8\left(F_{123}+F_{312}+F_{231}-G_{123}-G_{312}-G_{231}+H_{123}\right) A_{4}^{x}
$$

where

$$
\begin{aligned}
& F_{123}=(\lambda \bar{\lambda})^{5} Q\left(\lambda \gamma^{a d} r\right)\left(\lambda \gamma^{c e} r\right)\left(\lambda \gamma^{b f} r\right)\left(r \gamma^{a b} W^{1}\right)\left(r \gamma^{d e} W^{2}\right)\left(\bar{\lambda} \gamma^{c f} W^{3}\right) \\
& G_{123}=(\lambda \bar{\lambda})^{5} Q\left(\lambda \gamma^{c e} r\right)\left(\lambda \gamma^{b f} r\right)\left(\lambda \gamma^{a d} \bar{\lambda}\right)\left(r \gamma^{a b} W^{1}\right)\left(r \gamma^{d e} W^{2}\right)\left(r \gamma^{c f} W^{3}\right) \\
& H_{123}=(\lambda \bar{\lambda})^{6}\left(\lambda \gamma^{a d} r\right)\left(\lambda \gamma^{c e} r\right)\left(\lambda \gamma^{b f} r\right)\left(r \gamma^{a b} W^{1}\right)\left(r \gamma^{d e} W^{2}\right)\left(r \gamma^{c f} W^{3}\right)
\end{aligned}
$$

It is not difficult to show that $H_{123}$ is totally antisymmetric in [123] whereas $F_{123}$ and $G_{123}$ are antisymmetric in [12].

Let us rewrite the superfield $F_{123}$ using the Theorem 1. Since the $\gamma$-matrix traceless tensors are normalized such that $\mathcal{T}_{\alpha_{1} \ldots \alpha_{n}}^{\alpha_{1} \ldots \alpha_{n}}=1$, the factor $(\lambda \bar{\lambda})^{5}$ is inert under the application of the theorem and one can use (A.11) directly. Furthermore, all terms which still contain an explicit BRST charge after using (A.11) will be BRST-trivial because of the factor $(\lambda \bar{\lambda})^{6}$. So in fact only four terms in (A.11) are non-vanishing when applied to $F_{123}$. After straightforward algebra and discarding BRST-exact terms,

$$
\begin{aligned}
F_{123}=\frac{2}{33}( & \lambda \bar{\lambda})^{6}\left[-\left(\lambda \gamma^{a d} r\right)\left(\lambda \gamma^{c e} r\right)\left(\lambda \gamma^{b f} r\right)\left(r \gamma^{a b} W^{1}\right)\left(r \gamma^{d e} W^{2}\right)\left(r \gamma^{c f} W^{3}\right)\right. \\
& -\frac{1}{4}\left(\lambda \gamma^{d e} r\right)\left(\lambda \gamma^{a f} r\right)\left(\lambda \gamma^{b c} r\right)\left(r \gamma^{a b} W^{3}\right)\left(r \gamma^{c d} W^{1}\right)\left(r \gamma^{e f} W^{2}\right) \\
& \left.+\frac{1}{4}\left(\lambda \gamma^{d e} r\right)\left(\lambda \gamma^{a f} r\right)\left(\lambda \gamma^{b c} r\right)\left(r \gamma^{a b} W^{3}\right)\left(r \gamma^{c d} W^{2}\right)\left(r \gamma^{e f} W^{1}\right)\right]
\end{aligned}
$$

which implies that $F_{123}=-\frac{1}{11} H_{123}$ (and similarly $G_{123}=\frac{1}{11} H_{123}$ ). Plugging these results into (A.16) and taking into account the total antisymmetry of $H_{i j k}$ one finally obtains $L_{123}=\left(48^{3} 40 / 11\right) H_{123}$. Identical manipulations apply to $T_{12,3,4}$, so

$$
\begin{gathered}
L_{1234}^{m}(\lambda, \bar{\lambda}, r)=\frac{48^{3} 40}{11}(\lambda \bar{\lambda})^{6}\left(\lambda \gamma^{a f} r\right)\left(\lambda \gamma^{b c} r\right)\left(\lambda \gamma^{d e} r\right)\left(r \gamma^{a b} W^{1}\right)\left(r \gamma^{c d} W^{2}\right)\left(r \gamma^{e f} W^{3}\right) A_{4}^{m} \\
T_{12,3,4}(\lambda, \bar{\lambda}, r)=\frac{48^{3} 40}{11}(\lambda \bar{\lambda})^{6}\left(\lambda \gamma^{a f} r\right)\left(\lambda \gamma^{b c} r\right)\left(\lambda \gamma^{d e} r\right)\left(r \gamma^{a b} W_{12}\right)\left(r \gamma^{c d} W_{3}\right)\left(r \gamma^{e f} W_{4}\right)
\end{gathered}
$$

Similar manipulations can be used in $S_{1234}^{m}(\lambda, \bar{\lambda}, r)$ but for historic reasons we computed the five covariant derivatives before rewriting it with the factor $(\lambda \bar{\lambda})^{6}$. The resulting expression is not particularly illuminating and was therefore omitted. 


\section{Appendix B. Integration by parts}

Noting that one can replace $\Delta\left(z_{j} ; z_{k} ; z_{l}\right) X_{1 j}$ by $\Delta\left(z_{1} ; z_{k} ; z_{l}\right) X_{1 j}$ in (3.26) because $U_{i} U_{j} \sim$ $\left(d W_{i j}\right)\left(z_{j}\right) \eta_{i j}=\left(d W_{i j}\right)\left(z_{i}\right) \eta_{i j}$ it is straightforward to show that the identities which eliminate $X_{1 i}$ and $\bar{X}_{1 j}$ are given by

$$
\begin{aligned}
\Delta(1,3,4) \bar{\Delta}(2,3,4) X_{12} \bar{X}_{12}= & \Delta(1,3,4) \bar{\Delta}(1,3,4)\left(X_{23}+X_{24}\right)\left(\bar{X}_{23}+\bar{X}_{24}\right) \\
& +\Delta(1,3,4) \bar{\Delta}(2,3,4) s_{12} \tilde{\Omega}_{21}-\Delta(1,2,4) \bar{\Delta}(1,3,4) s_{23} \tilde{\Omega}_{32} \\
& +\Delta(1,2,3) \bar{\Delta}(1,3,4) s_{24} \tilde{\Omega}_{42} \\
\Delta(1,3,4) \bar{\Delta}(1,2,4) X_{12} \bar{X}_{13}= & \left(X_{23}+X_{24}\right)\left(-\bar{X}_{23}+\bar{X}_{34}\right) \Delta(1,3,4) \bar{\Delta}(1,2,4) \\
& +s_{23} \tilde{\Omega}_{23} \Delta(1,3,4) \bar{\Delta}(1,2,4), \\
\Delta(1,3,4) \bar{\Delta}(1,2,4) X_{12} \bar{X}_{23}= & {\left[\left(X_{23}+X_{24}\right) \bar{X}_{23}-s_{23} \tilde{\Omega}_{23}\right] \Delta(1,3,4) \bar{\Delta}(1,2,4) } \\
\Delta(1,3,4) \bar{\Delta}(1,2,3) X_{12} \bar{X}_{34}= & \left(X_{23}+X_{24}\right) \bar{X}_{34} \Delta(1,3,4) \bar{\Delta}(1,2,3),
\end{aligned}
$$

where we used that

$$
\bar{\partial}_{i} X_{j i}=s_{i j} \tilde{\Omega}_{j i}, \quad \partial_{i} \bar{X}_{j i}=s_{i j} \tilde{\Omega}_{i j}, \quad \bar{\partial}_{i} X_{i j}=-s_{i j} \tilde{\Omega}_{j i}, \quad \partial_{i} \bar{X}_{i j}=-s_{i j} \tilde{\Omega}_{i j},
$$

and defined $\tilde{\Omega}_{i j}=\left(\alpha^{\prime} / 2\right) \pi \Omega\left(z_{i}, z_{j}\right)$. All other identities needed to write $|\mathcal{F}|^{2}$ in a basis of integrals follow from the above by relabeling. Applying them together with

$$
\tilde{\Omega}_{i j} \Delta(j, k, l) \bar{\Delta}(i, k, l)=\tilde{\Omega}_{j i} \Delta(i, k, l) \bar{\Delta}(j, k, l),
$$

implies that $|\mathcal{F}|^{2}$ is equal to

$$
\begin{aligned}
& +\left|C_{32,1,4}\right|^{2}\left(X_{23} \bar{X}_{23}-s_{23} \tilde{\Omega}_{23}\right) \Delta(1,3,4) \bar{\Delta}(1,2,4) \\
& -\left|C_{24,1,3}\right|^{2}\left(X_{24} \bar{X}_{24}-s_{24} \tilde{\Omega}_{24}\right) \Delta(1,3,4) \bar{\Delta}(1,2,3) \\
& +\left|C_{34,1,2}\right|^{2}\left(X_{34} \bar{X}_{34}-s_{34} \tilde{\Omega}_{34}\right) \Delta(1,2,4) \bar{\Delta}(1,2,3) \\
& +C_{32,1,4} \tilde{C}_{24,1,3} X_{23} \bar{X}_{24} \Delta(1,2,4) \bar{\Delta}(1,3,4)+C_{32,1,4} \tilde{C}_{34,1,2} X_{23} \bar{X}_{34} \Delta(1,3,4) \bar{\Delta}(1,2,4) \\
& +C_{24,1,3} \tilde{C}_{32,1,4} X_{24} \bar{X}_{23} \Delta(1,3,4) \bar{\Delta}(1,2,4)+C_{24,1,3} \tilde{C}_{34,1,2} X_{24} \bar{X}_{34} \Delta(1,3,4) \bar{\Delta}(1,2,3) \\
& +C_{34,1,2} \tilde{C}_{32,1,4} X_{34} \bar{X}_{23} \Delta(1,2,4) \bar{\Delta}(1,3,4)+C_{34,1,2} \tilde{C}_{24,1,3} X_{34} \bar{X}_{24} \Delta(1,2,4) \bar{\Delta}(1,3,4) \\
& +s_{12} \tilde{\Omega}_{12}\left|M_{12,3,4}\right|^{2} \Delta(2,3,4) \bar{\Delta}(1,3,4)-s_{13} \tilde{\Omega}_{13}\left|M_{13,2,4}\right|^{2} \Delta(2,3,4) \bar{\Delta}(1,2,4) \\
& +s_{14} \tilde{\Omega}_{14}\left|M_{14,2,3}\right|^{2} \Delta(2,3,4) \bar{\Delta}(1,2,3)+s_{23} \tilde{\Omega}_{23}\left|M_{23,1,4}\right|^{2} \Delta(1,3,4) \bar{\Delta}(1,2,4) \\
& -s_{24} \tilde{\Omega}_{24}\left|M_{24,1,3}\right|^{2} \Delta(1,3,4) \bar{\Delta}(1,2,3)+s_{34} \tilde{\Omega}_{34}\left|M_{34,1,2}\right|^{2} \Delta(1,2,4) \bar{\Delta}(1,2,3)
\end{aligned}
$$


where we defined (the others follow from relabeling)

$$
C_{24,1,3} \equiv M_{24,1,3}+M_{14,2,3}+M_{12,3,4}
$$

The $\alpha^{\prime}$-expansion of the above integrals has not been derived but one can argue from the results of [42] that $\eta_{i j} \bar{\eta}_{i j}-\tilde{\Omega}_{i j} s_{i j}^{-1}$ and $\eta_{i j} \bar{\eta}_{i k}$ have no kinematic poles. Therefore the leading-order contribution from (B.4) is given by the $\tilde{\Omega}_{i j}$ terms and it follows from relabeling of integration variables that they are all equal to $\pm \int \tilde{\Omega}_{12} \Delta(2,3,4) \bar{\Delta}(1,3,4)$ (the sign is easy to obtain). Thus the low-energy limit of $|\mathcal{F}|^{2}$ in $(3.25)$ corresponds to ${ }^{10}$

$$
-\frac{\sqrt{2} \pi^{7}}{2^{23} 3^{7}} \kappa^{4} e^{4 \lambda}\left(\frac{\alpha^{\prime}}{2}\right)^{7} \int \frac{d^{2} \Omega_{I J}}{Z_{3}^{-10}} \int \prod_{i=1}^{4} d^{2} z_{i} \Omega_{12} \Delta(2,3,4) \bar{\Delta}(1,3,4)\langle\mathcal{K}\rangle_{(-3)}\left\langle\prod_{j=1}^{4} e^{i k^{j} \cdot x^{j}}\right\rangle
$$

where we used $\tilde{\Omega}_{12}=\pi\left(\alpha^{\prime} / 2\right) \Omega_{12}$ and defined,

$$
\mathcal{K}=\frac{\left|T_{23,1,4}\right|^{2}}{s_{23}}+\frac{\left|T_{24,1,3}\right|^{2}}{s_{24}}+\frac{\left|T_{34,1,2}\right|^{2}}{s_{34}}+\frac{\left|T_{12,3,4}\right|^{2}}{s_{12}}+\frac{\left|T_{13,2,4}\right|^{2}}{s_{13}}+\frac{\left|T_{14,2,3}\right|^{2}}{s_{14}} .
$$

\section{B.1 Open superstring}

In the case of the open superstring it is not difficult to argue that the corresponding low-energy limit is

$$
\mathcal{K}^{\text {(open) }}=\frac{T_{23,4,1}+T_{41,2,3}}{s_{23}}+\frac{T_{34,1,2}+T_{12,3,4}}{s_{34}} .
$$

The component expansion of (B.8) provides a good consistency check for the methods of [25] since one recovers the $\alpha^{\prime 3}$ interaction of the open superstring tree-level amplitude [43],

$$
\frac{\left\langle T_{23,4,1}+T_{41,2,3}\right\rangle}{s_{23}}+\frac{\left\langle T_{34,1,2}+T_{12,3,4}\right\rangle}{s_{34}}=1344 \cdot 40 \cdot 48^{3} \cdot 2880 A_{1234}^{\mathrm{YM}} s_{12} s_{13} s_{23} .
$$

10 The minus sign compensates the "convention" $i k^{m} \rightarrow k^{m}$ in the Koba-Nielsen factor. 


\section{References}

[1] N. Berkovits, "Super-Poincare covariant quantization of the superstring," JHEP 0004, 018 (2000) [arXiv:hep-th/0001035].

[2] N. Berkovits, "Pure spinor formalism as an $\mathrm{N}=2$ topological string," JHEP 0510, 089 (2005) [arXiv:hep-th/0509120].

[3] E. D'Hoker and D.H. Phong, "Two-Loop Superstrings VI: Non-Renormalization Theorems and the 4-Point Function," Nucl. Phys. B 715, 3 (2005) [arXiv:hep-th/0501197].

[4] N. Berkovits, "Super-Poincare covariant two-loop superstring amplitudes," JHEP 0601, 005 (2006) [arXiv:hep-th/0503197].

[5] N. Berkovits and C.R. Mafra, "Equivalence of two-loop superstring amplitudes in the pure spinor and RNS formalisms," Phys. Rev. Lett. 96, 011602 (2006) [arXiv:hepth/0509234].

[6] N. Berkovits and N. Nekrasov, "Multiloop superstring amplitudes from non-minimal pure spinor formalism," JHEP 0612, 029 (2006) [arXiv:hep-th/0609012].

[7] N. Berkovits, "New higher-derivative R**4 theorems," Phys. Rev. Lett. 98, 211601 (2007). [arXiv:hep-th/0609006].

[8] E. Witten, "More On Superstring Perturbation Theory," [arXiv:1304.2832 [hep-th]].

[9] Y. Aisaka and N. Berkovits, "Pure Spinor Vertex Operators in Siegel Gauge and Loop Amplitude Regularization," JHEP 0907, 062 (2009). [arXiv:0903.3443 [hep-th]].

[10] H. Gomez, "One-loop Superstring Amplitude From Integrals on Pure Spinors Space," JHEP 0912, 034 (2009) [arXiv:0910.3405 [hep-th]].

[11] H. Gomez, C.R. Mafra, "The Overall Coefficient of the Two-loop Superstring Amplitude Using Pure Spinors," JHEP 1005, 017 (2010). [arXiv:1003.0678 [hep-th]].

[12] M.B. Green and P. Vanhove, "Duality and higher derivative terms in M theory," JHEP 0601, 093 (2006). [arXiv:hep-th/0510027].

[13] E.P. Verlinde and H.L. Verlinde, "Chiral bosonization, determinants and the string partition function," Nucl. Phys. B 288, 357 (1987).

[14] E. D'Hoker and D.H. Phong, "The Geometry of String Perturbation Theory," Rev. Mod. Phys. 60, 917 (1988).

[15] W. Siegel, "Classical Superstring Mechanics," Nucl. Phys. B263, 93 (1986).

[16] I. Oda and M. Tonin, "Y-formalism and $b$ ghost in the Non-minimal Pure Spinor Formalism of Superstrings," Nucl. Phys. B 779, 63 (2007) [arXiv:0704.1219 [hep-th]].

[17] R. Lipinski Jusinskas, "Nilpotency of the $\mathrm{b}$ ghost in the non-minimal pure spinor formalism," JHEP 1305, 048 (2013). [arXiv:1303.3966 [hep-th]].

[18] E.Witten, "Twistor-Like Transform In Ten-Dimensions" Nucl.Phys. B 266, 245 (1986)

[19] E. D'Hoker, M. Gutperle and D.H. Phong, "Two-loop superstrings and S-duality," Nucl. Phys. B 722, 81 (2005) [arXiv:hep-th/0503180]. 
[20] C.R. Mafra, O. Schlotterer, S. Stieberger and D. Tsimpis, "A recursive method for SYM n-point tree amplitudes," Phys. Rev. D 83, 126012 (2011). [arXiv:1012.3981 [hep-th]].

[21] M.B. Green, J.G. Russo and P. Vanhove, "Low energy expansion of the fourparticle genus-one amplitude in type II superstring theory," JHEP 0802, 020 (2008) [arXiv:0801.0322 [hep-th]].

[22] Griffiths and Harris, "Principles of Algebraic Geometry", [Wiley Classics Library Edition Published 1994]

[23] C.L. Siegel, "Symplectic Geometry", Am. J. Math. 65 (1943) 1-86;

[24] N. Berkovits, "Explaining pure spinor superspace," [arXiv:hep-th/0612021].

[25] C.R. Mafra, "PSS: A FORM Program to Evaluate Pure Spinor Superspace Expressions," [arXiv:1007.4999 [hep-th]].

[26] J.A.M. Vermaseren, "New features of FORM," [arXiv:math-ph/0010025].

[27] H. Gomez and C.R. Mafra, work in progress.

[28] C.R. Mafra and O. Schlotterer, "The Structure of n-Point One-Loop Open Superstring Amplitudes," [arXiv:1203.6215 [hep-th]].

[29] N. Berkovits, M.B. Green, J.G. Russo and P. Vanhove, "Non-renormalization conditions for four-gluon scattering in supersymmetric string and field theory," JHEP 0911, 063 (2009). [arXiv:0908.1923 [hep-th]].

[30] E. D'Hoker and M. B. Green, "Zhang-Kawazumi Invariants and Superstring Amplitudes," [arXiv:1308.4597 [hep-th]].

[31] O. Schlotterer and S. Stieberger, "Motivic Multiple Zeta Values and Superstring Amplitudes," [arXiv:1205.1516 [hep-th]].

[32] M.B. Green and M. Gutperle, "Effects of D instantons," Nucl. Phys. B 498, 195 (1997). [hep-th/9701093]. ;

M.B. Green, M. Gutperle and P. Vanhove, "One loop in eleven-dimensions," Phys. Lett. B 409, 177 (1997). [hep-th/9706175].

[33] M.B. Green, H.-h. Kwon and P. Vanhove, "Two loops in eleven-dimensions," Phys. Rev. D 61, 104010 (2000). [hep-th/9910055].

[34] N. Berkovits and C.R. Mafra, "Some superstring amplitude computations with the non-minimal pure spinor formalism," JHEP 0611, 079 (2006) [arXiv:hep-th/0607187].

[35] C.R. Mafra and C. Stahn, "The One-loop Open Superstring Massless Five-point Amplitude with the Non-Minimal Pure Spinor Formalism," JHEP 0903, 126 (2009) [arXiv:0902.1539 [hep-th]].

[36] J. Hoogeveen and K. Skenderis, "Decoupling of unphysical states in the minimal pure spinor formalism I," JHEP 1001, 041 (2010). [arXiv:0906.3368 [hep-th]].

[37] Christian Stahn, private communication in 2008

[38] N. Berkovits and N. Nekrasov, "The Character of pure spinors," Lett. Math. Phys. 74, 75 (2005). [arXiv:hep-th/0503075]. 
[39] Y. Aisaka, E.A. Arroyo, N. Berkovits and N. Nekrasov, "Pure Spinor Partition Function and the Massive Superstring Spectrum," JHEP 0808, 050 (2008) [arXiv:0806.0584 [hep-th]].

[40] N. Berkovits, "Multiloop amplitudes and vanishing theorems using the pure spinor formalism for the superstring," JHEP 0409, 047 (2004) [arXiv:hep-th/0406055].

[41] C. Stahn, "Fermionic superstring loop amplitudes in the pure spinor formalism," JHEP 0705, 034 (2007). [arXiv:0704.0015 [hep-th]].

[42] M.B. Green, C.R. Mafra and O. Schlotterer, "Multiparticle one-loop amplitudes and S-duality in closed superstring theory," [arXiv:1307.3534 [hep-th]].

[43] C.R. Mafra, O. Schlotterer and S. Stieberger, "Complete N-Point Superstring Disk Amplitude I. Pure Spinor Computation," Nucl. Phys. B 873, 419 (2013). [arXiv:1106.2645 [hep-th]]. ;

C.R. Mafra, O. Schlotterer and S. Stieberger, "Complete N-Point Superstring Disk Amplitude II. Amplitude and Hypergeometric Function Structure," Nucl. Phys. B 873, 461 (2013). [arXiv:1106.2646 [hep-th]]. 